\title{
INTERNATIONAL JOURNAL OF MODERN PHYSICS B
}

Article ID: JPB20076408

Accepted April $22^{\text {nd }} 2020$

\section{ADOMAIN COMPUTATION OF RADIATIVE-CONVECTIVE BI-DIRECTIONAL STRETCHING FLOW OF A MAGNETIC NON-NEWTONIAN FLUID IN POROUS MEDIA WITH HOMOGENEOUS- HETEROGENEOUS REACTIONS}

\author{
S.R. Mishra ${ }^{1}$, MD. Shamshuddin ${ }^{2 *}$, O. Anwar Bég ${ }^{3}$ and Ali Kadir ${ }^{3}$ \\ ${ }^{1}$ Department of Mathematics, Siksha O Anusandhan Deemed to be University, Bhubaneswar, India. \\ ${ }^{2}$ Department of Mathematics, Vaagdevi College of Engineering, Warangal, India. \\ ${ }^{3}$ Department of Aeronautical and Mechanical Engineering, University of Salford, Manchester, UK. \\ *Corresponding author-Email: shammaths@gmail.com
}

\begin{abstract}
In the present communication, laminar, incompressible, hydromagnetic flow of an electrically conducting non-Newtonian (Sisko) fluid over a bi-directional stretching sheet in a porous medium is studied theoretically. Thermal radiation flux, homogeneous-heterogeneous chemical reactions and convective wall heating are included in the model. Darcy's model is employed for the porous medium and Rosseland's model for radiation heat transfer. The governing partial differential equations for mass, momentum, energy and concentration are reduced into ordinary differential equations via similarity transformations. The resultant nonlinear ordinary differential equations with transformed boundary conditions are then solved via the semi-analytical Adomain decomposition method (ADM). Validation with earlier studies is included for the non-radiative case. Extensive visualization of velocity, temperature and species concentration distributions for various emerging parameters is included. Increasing magnetic field and inverse permeability parameter are observed to decelerate both the primary and secondary velocity magnitudes whereas they increase temperatures in the regime. Increasing sheet stretching ratio weakly accelerates the primary flow throughout the boundary layer whereas it more dramatically accelerates the secondary flow near sheet surface. Temperature is consistently reduced with increasing stretching sheet ratio whereas it is strongly enhanced with greater radiative parameter. With greater Sisko non-Newtonian power-law index the primary velocity and temperature are decreased whereas the secondary velocity is increased. Increasing both homogenous and heterogenous chemical reaction parameters is found to weakly and more strongly, respectively, deplete concentration magnitudes whereas greater Schmidt number enhances them. Primary and secondary skin friction and Nusselt number profiles are also computed. The study is relevant to electro-conductive (magnetic polymer) materials processing operations.
\end{abstract}

KEYWORDS: Sisko fluid, homogeneous-heterogenous reactions, porous medium, thermal radiation, ADM, bi-axial stretching, thermal magnetic polymer processing.

\section{INTRODUCTION}

Magnetic polymers are being increasingly deployed in modern engineering applications. Such materials combine magnetohydrodynamic and rheological properties and can be customized for different systems including power-generation [1], smart coating technologies [2,3], 
supercapacitor electrodes [4] and solar collector receiver surface enhancement [5]. The use of advanced multi-physical fluid dynamics models can significantly improve fabrication processes for such materials and leads to an optimization of quality control, in particular for heat and mass transfer. With judicious use of an applied magnetic field the polymer constitution and performance can be manipulated. Since magnetic polymers exhibit non-Newtonian properties it is essential to utilize appropriate constitutive models which correctly characterize the rheology of such fluids. Conventional Newtonian models are inadequate. Engineers have therefore explored many different rheological models to predict more closely the shear-stress strain behavior of magnetic polymers. Examples of models employed include fractional Burgers viscoelastic models [6], Eringen micropolar models [7] and couple stress nanofluid models [8]. The Sisko model [9] is a relatively simple non-Newtonian model which provides robust predictions for high shear rate flows and is a modification of the power-law model. Originally introduced for lubricants, the Sisko model has been adopted in a wide spectrum of applications including materials processing, biomechanics and thermal engineering systems. These applications include heat and mass transfer and employ a number of numerical approaches to solve nonlinear boundary value problems. Malik et al. [10] used a Runge-KuttaFehlberg method to compute thermal conductivity effects on velocity and temperature characteristics in Sisko fluid dissipative boundary layer flow from a stretching cylindrical body. Khan et al. [11] studied the hydromagnetic Sisko flow in a pipe annulus considering both shearthinning and thickening behavior. Khan et al. [12] deployed both homotopy and finite difference methods to simulate thermal transport in Sisko fluids in an annulus. El-Dabe et al. [13] studied the magnetic pumping of Sisko fluid in a porous conduit under peristaltic wave motion. Zaman et al. [14] computed the time-dependent blood flow in a cylindrical vessel using the Sisko model. Khan et al. [15] used the homotopy analysis method (HAM) to compute the influence of rheological power-law index, Sisko material parameter and stretching parameter on the radially stretching flow of a Sisko liquid. Munir et al. [16] examined the impact of forced convection on stretching sheet flow of a Sisko fluid.

In many materials processing operations (including magnetic polymer synthesis), chemical reactions arise. There are two major classifications of chemical reactions, namely homogeneous and heterogeneous. Chemical changes occurring with liquids or gases depend on the type of interactions of these chemical substances. Homogeneous reactions occur in one phase only whereas heterogeneous reactions occur in two or more phases. Chemically reactive materials processing often features purely heterogeneous chemical reactions (catalytic 
techniques) and also homogeneous-heterogeneous reactions (magnetic materials, corrosive processes, ceramics, polymers etc). Recently a number of investigators have addressed chemically reactive non-Newtonian and magnetized flows. Shamshuddin et al. [17] used a finite element code to simulate double-diffusive convection in reactive micropolar flow. Rajesh et al. [18] studied homogenous reaction effects on oscillating magnetic boundary layer flow from a curved surface. Doh et al. [19] employed a homotopy method to study the impact of homogeneous and heterogeneous reactions on hydromagnetic silver-water nanofluid Von Karman swirling flow, heat and mass transfer. Nadeem et al. [20] analysed ferrofluid rheological flow from a stretching cylinder with magnetization and homogeneous and heterogeneous reactions. Further studies of non-Newtonian reactive flows include Ravi Kiran et al. [21] (who employed a micropolar model), Alshomrani and Ullah [22] (who considered carbon nanotube reactive fluid flow from a bi-directional stretching sheet) and Abbas and Sheikh [23] (who studied stagnation ferrofluid flow with homogeneous-heterogeneous reactions). These studies have generally shown that there is a considerable deviation between solutions for higher order chemical effects compared with lower order chemical reaction models.

In high-temperature fabrication of polymers [24, 25], radiative heat transfer arises in addition to thermal conduction and thermal convection. To simulate radiative flux many approaches are available. The most general is the solution of the integro-differential equation of radiative heat transfer. This furnishes detailed information on radiative behavior at different spectra and wavelengths. However, it is very challenging computationally. When other effects are present e.g. rheology, magnetohydrodynamics etc, simpler approaches are more pragmatic and these usually feature an algebraic flux approximation which may be (in progressively increasing complexity) of the Rosseland type, Milne-Eddington type, Schuster-Schwartzchild type, Traugott P1 flux model, Hamaker six flux model etc. In multi-physical coating flows, the Rosseland model has been shown to be reasonably accurate for high optical thicknesses. Although it does not allow optical thickness or spectral effects to be simulated, it does provide a mechanism for evaluating the relative role of thermal conduction and thermal radiation flux. Several researchers have studied radiative heat transfer in materials processing including Lu et al. [26] (magnetic non-Newtonian reactive polymer flows), Kumpaty and Roux [27] (glass synthesis), Rozzi et al. [28] (laser-processing of ceramics) and Bég et al. [29] (dissipative magnetic coating flows). 
In the present article, motivated by providing more comprehensive insight into magnetic polymer processing at high temperature, a mathematical model is developed for radiative magnetohydrodynamic Sisko fluid flow from a bidirectional stretching embedded in a Darcian porous medium with homogeneous and heterogeneous reactions. This model extends the earlier study of Hayat et al. [30] to consider radiative heat transfer and also porous medium drag effects. The partial differential boundary value problem is converted to a nonlinear dimensionless ordinary differential boundary value problem with appropriate similarity transformations. A robust solution is obtained with the semi-analytical Adomain decomposition method (ADM) [31] and validation with the non-radiative case [30] is included. A detailed parametric study of the influence of magnetic, Darcy (permeability), stretching sheet ratio, Sisko non-Newtonian power-law index, Schmidt number and homogenous and heterogenous chemical reaction parameters on velocity, temperature and species concentration distributions is conducted with graphical visualization of results. included. Primary and secondary skin friction and Nusselt number profiles are also computed. The computations may be beneficial in further understanding the complex interplay between chemical reactions, magnetic field and rheology in materials processing operations.

\section{MATHEMATICAL REACTIVE MAGNETIC POL YMER STRETCHING FLOW MODEL}

Consider the flow of an incompressible electrically conducting polymeric Sisko fluid with heat and mass transfer from a bi-directional stretching sheet under a transverse (vertical) magnetic field of strength $B_{0}$ adjacent to a porous medium. Hall and electric field effects are ignored. The porous medium is assumed to isotropic and homogenous and Darcy's model is employed for viscous-dominated low Reynolds number transport. We consider a Cartesian coordinate system such that the sheet coincides with the $x y$-plane and fluid occupies the space $z \geq 0$ as illustrated in Figure.1. Let $U_{w}(x)=c x$ and $V_{w}(x)=d y$ denote the surface stretching velocities along the $x, y$ directions and $c, d>0$. The temperature at the sheet is governed by a convective heating process which is described by a heat transfer coefficient $h_{f}$ and temperature of the hot fluid $T_{f}$ below the sheet. Viscous dissipation effects are ignored. The corresponding equations, under the above assumptions, describing the three-dimensional Sisko fluid motion are given by extending the model of Hayat et al. [30] with Darcian drag force and radiative heat flux terms, leading to: 
$\frac{\partial u}{\partial x}+\frac{\partial v}{\partial y}+\frac{\partial w}{\partial z}=0$

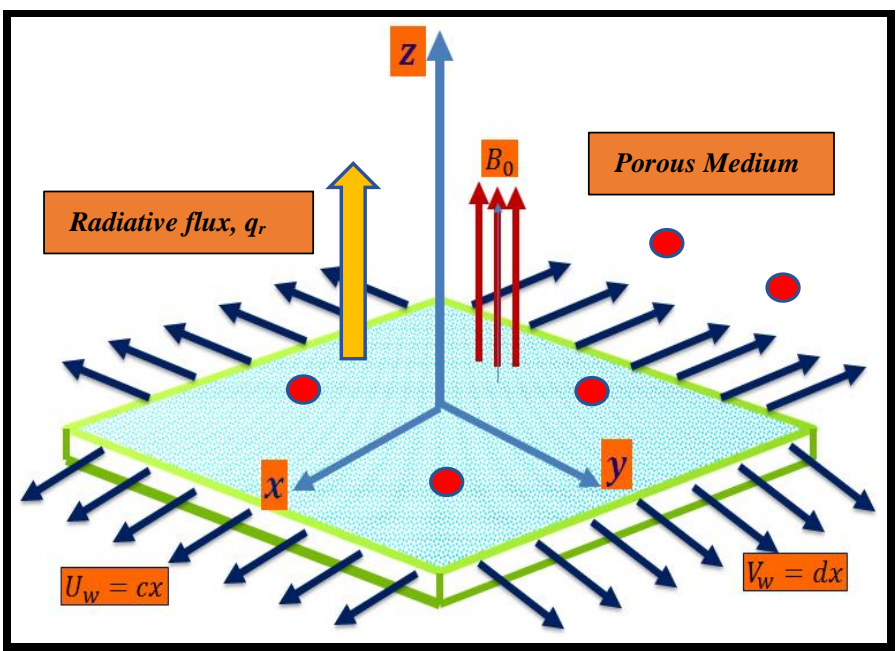

Figure 1: Schematic physical model for bi-directional stretching flow of a Sisko fluid

$$
\begin{aligned}
& u \frac{\partial u}{\partial x}+v \frac{\partial u}{\partial y}+w \frac{\partial u}{\partial z}=\frac{a}{\rho} \frac{\partial^{2} u}{\partial z^{2}}-\frac{b}{\rho} \frac{\partial}{\partial z}\left(-\frac{\partial u}{\partial z}\right)^{n}-\frac{\mu}{k} u-\frac{\sigma B_{0}^{2}}{\rho} u, \\
& u \frac{\partial v}{\partial x}+v \frac{\partial v}{\partial y}+w \frac{\partial v}{\partial z}=\frac{a}{\rho} \frac{\partial^{2} v}{\partial z^{2}}+\frac{b}{\rho} \frac{\partial}{\partial z}\left(-\frac{\partial u}{\partial z}\right)^{n-1} \frac{\partial v}{\partial z}-\frac{\mu}{k} v-\frac{\sigma B_{0}^{2}}{\rho} v, \\
& u \frac{\partial T}{\partial x}+v \frac{\partial T}{\partial y}+w \frac{\partial T}{\partial z}=\alpha_{m} \frac{\partial^{2} T}{\partial z^{2}}-\frac{1}{\rho C_{p}} \frac{\partial q_{r}}{\partial z}, \\
& u \frac{\partial a_{1}}{\partial x}+v \frac{\partial a_{1}}{\partial y}+w \frac{\partial a_{1}}{\partial z}=D_{A} \frac{\partial^{2} a_{1}}{\partial z^{2}}-k_{c} a_{1} b_{1}^{2}, \\
& u \frac{\partial b_{1}}{\partial x}+v \frac{\partial b_{1}}{\partial y}+w \frac{\partial b_{1}}{\partial z}=D_{B} \frac{\partial^{2} b_{1}}{\partial z^{2}}+k_{c} a_{1} b_{1}^{2},
\end{aligned}
$$

The appropriate boundary conditions for the regime are:

at $z=0: u=U_{w}=c x, v=V_{w}=d y, w=0,-k \frac{\partial T}{\partial z}=h_{f}\left(T_{f}-T\right), D_{A} \frac{\partial a_{1}}{\partial z}=k_{s} a_{1}, D_{B} \frac{\partial b_{1}}{\partial z}=-k_{s} a_{1}$

at $z \rightarrow \infty: u \rightarrow 0, \quad v \rightarrow 0, \quad T \rightarrow T_{\infty}, \quad a_{1} \rightarrow a_{0}, \quad b_{1} \rightarrow 0$

A simple model of heterogeneous-homogeneous reactions is adopted in Eqns. (5) and (6) [32]

$A_{1}+2 A_{2} \rightarrow 3 A_{2}$, rate $=k_{c} a_{1} b_{1}^{2}$

Here the catalyst surface heterogeneous reaction is defined as follows:

$A_{1} \rightarrow A_{2}$, rate $=k_{s} a_{1}$ 
Here the chemical species $A_{1}$ and $A_{2}$ have concentrations $a_{1}$ and $b_{1}$ respectively. $k_{c}$ and $k_{s}$ are the rate constants. In Eqns. (1)-(7) $u, v$ and $w$ designate the velocity components, $\rho$ denotes base fluid density, $D_{A}$ and $D_{B}$ are the respective diffusion species coefficients, $a_{0}$ is the positive dimensionless constant, $T$ denotes the surface fluid temperature, $\alpha_{m}=\kappa / \rho C_{p}$ represents thermal diffusivity, $\kappa$ the thermal conductivity of the magnetic polymer (Sisko fluid), $\rho C_{p}$ heat capacity of fluid, $a$ and $b$ denote material parameters of the Sisko fluid and $n$ is the Sisko power-law rheological index ( $n>0$ characterizes the non-Newtonian features of the fluid). $q_{r}$ signifies radiative heat flux which is assumed from [33] and is denoted in the Rosseland model [29] as $\frac{\partial q_{r}}{\partial z}=-\frac{16 \sigma^{*}}{3 k^{*}} \frac{\partial}{\partial z}\left(T^{3} \frac{\partial T}{\partial z}\right)$. It should be noted that unlike classical cases, the nonlinear form of thermal radiation is considered here. Furthermore, the nonlinear thermal radiation effect in the linearized Rosseland approximation is a simple rescaling of the Prandtl number by a factor involving the radiation parameter. The solution of the radiation problem in the linearized Rosseland approximation does not require any additional numerical or analytical efforts. It is seen that the energy equation in case of nonlinear radiation is strongly nonlinear and contains an additional temperature parameter which is the ratio of wall and ambient temperature. To facilitate a numerical solution of the boundary value problem, it is judicious to introduce the following dimensionless variables [34-35]:

$$
\begin{aligned}
& u=c x f^{\prime}(\eta), v=d g^{\prime}(\eta), w=-c\left(\frac{c^{n-2}}{\rho / b}\right)^{1 /(n+1)}\left(\frac{2 n}{n+1} f+\frac{1-n}{n+1} \eta f^{\prime}+g\right) x^{\frac{n-1}{n+1}}, \\
& \left.\theta(\eta)=\frac{T-T_{\infty}}{T_{f}-T_{\infty}}, \quad a_{1}=a_{0} h(\eta), \quad b_{1}=a_{0} h(\eta), \quad \eta=z\left(\frac{c^{2-n}}{\rho / b}\right)^{1 / n+1} x^{\frac{1-n}{1+n}}\right\}
\end{aligned}
$$

Implementing Eqn. (10) in Eqns. (2)-(7) produces the following dimensionless similarity equations in which equation (1) is automatically satisfied:

$$
\begin{aligned}
& A f^{\prime \prime \prime}+n\left(-f^{\prime \prime}\right)^{n-1} f^{\prime \prime \prime}+\left(\frac{2 n}{n+1}\right) f f^{\prime \prime}-\left(-f^{\prime}\right)^{2}+g f^{\prime \prime}-K f^{\prime}-M^{2} f^{\prime}=0 \\
& A g^{\prime \prime \prime}+\left(-f^{\prime \prime}\right)^{n-1} g^{\prime \prime \prime}-(n-1)\left(-f^{\prime \prime}\right)^{n-2} f^{\prime \prime \prime} g^{\prime \prime}+\left(\frac{2 n}{n+1}\right) f g^{\prime \prime}-\left(-g^{\prime}\right)^{2}+g g^{\prime \prime}-K g^{\prime}-M^{2} g^{\prime}=0 \\
& {\left[\left\{1+R\left(1+\left(\theta_{w}-1\right) \theta\right)^{3}\right\} \theta^{\prime}\right]^{\prime}+\operatorname{Pr}\left(\frac{2 n}{n+1}\right) f \theta^{\prime}+\operatorname{Pr} g \theta^{\prime}=0} \\
& \phi^{\prime \prime}+S c\left(\frac{2 n}{n+1}\right) f \phi^{\prime}+S c g \phi^{\prime}-S c k_{1} h^{2} \phi=0
\end{aligned}
$$


$\delta_{1} h^{\prime \prime}+S c\left(\frac{2 n}{n+1}\right) f h^{\prime}+S c g h^{\prime}+S c k_{1} h^{2} \phi=0$

$f=0, g=0, f^{\prime}=1, g^{\prime}=\alpha, \theta^{\prime}=-\gamma(1-\theta), \phi^{\prime}=k_{2} \phi, \delta_{1} h^{\prime}=-k_{2} \phi$ at $\eta=0$

$f^{\prime} \rightarrow 0, g^{\prime} \rightarrow 0, \theta \rightarrow 0, \phi \rightarrow 0, h \rightarrow 0$ when $\eta \rightarrow \infty$

The dimensionless flow parameters featured in Eqns. (11)-(16) are defined illustrated as below:

$$
\begin{aligned}
& A=\frac{\operatorname{Re}_{b}^{2 / n+1}}{\operatorname{Re}_{a}}, \operatorname{Re}_{a}=\frac{\rho x U_{w}}{a}, \operatorname{Re}_{b}=\frac{\rho x^{n} U_{w}^{2-n}}{b}, \quad K=\frac{\mu}{k U_{w}} x, M^{2}=\frac{\sigma B_{0}^{2}}{\rho c}, \\
& \alpha=\frac{d}{c}, \quad k_{1}=\frac{k_{c} a_{0}^{2}}{c}, \quad k_{2}=\frac{k_{S} c x}{D_{A} z} \mathrm{Re}_{b}^{-1 /(n+1)}, \quad \gamma=\frac{h_{f}}{k} x \operatorname{Re}_{b}^{-1 /(n+1)}, \delta_{1}=\frac{D_{A}}{D_{B}}, \\
& \operatorname{Pr}=\frac{x U_{w}}{\alpha_{m}} \operatorname{Re}_{b}^{-2 /(n+1)}, R=\frac{16 \sigma^{*}}{3 k k^{*}} T_{\infty}^{3}, \theta_{w}=\frac{T_{f}}{T_{\infty}}, \quad S c=\frac{U_{w} x}{D_{A}} \operatorname{Re}_{b}^{-2 /(n+1)},
\end{aligned}
$$

Here $A$ signifies the Sisko rheological fluid material parameter, $\operatorname{Re}_{a}$ and $\operatorname{Re}_{b}$ are local Reynolds numbers, $K$ indicates inverse permeability parameter, $M$ indicates the magnetic body force parameter, $\alpha$ designates the stretching sheet ratio parameter, $k_{1}$ and $k_{2}$ define the intensity (strength) of the homogenous and heterogeneous reactions respectively, $\operatorname{Pr}$ denotes Prandtl number, $R$ denotes thermal radiation parameter, $S c$ denotes Schmidt number, $\delta_{1}$ represents the diffusion coefficient ratio, $\gamma$ denotes generalized Biot number. It is noted that an additional temperature ratio parameter $\theta_{w}$ arises and due to this reason the energy equation will be more nonlinear; by using $q_{r}=-\frac{16 \sigma^{*}}{3 k^{*}} T^{3} \frac{\partial T}{\partial z}$ in Eqn. (4) leads to the more appropriate form i.e. Eqn. (13). The similarity solutions are possible only in the case $n=1$ (for viscous fluid) and the parameters $A=0$ and $\gamma$ are independent of x, i.e. $\gamma$ is constant. Herein local similar solutions are developed since in general the parameters in the derived model are functions of the independent variable $x$ ([36-37]. The coefficients of diffusion of chemical species $A_{1}$ and $A_{2}$ may be taken to be the same so that the diffusion coefficients $D_{A}$ and $D_{B}$ are identical i.e. $\delta_{1}=1$ and thus the following relation will be obtained:

$\phi(\eta)+h(\eta)=1$

Eqns. (14) and (15) may therefore be combined to yield the following single concentration equation [30]:

$\phi^{\prime \prime}+S c\left(\frac{2 n}{n+1}\right) f \phi^{\prime}+S c g \phi^{\prime}-S c k_{1} \phi(1-\phi)^{2}=0$,

The associated concentration boundary conditions: 
$\phi^{\prime}(0)=k_{2} \phi(0)$ as $\phi(\infty) \rightarrow 1$

The final transformed coupled ordinary differential equations with boundary conditions emerge as follows:

$$
\begin{aligned}
& A f^{\prime \prime \prime}+n\left(-f^{\prime \prime}\right)^{n-1} f^{\prime \prime \prime}+\left(\frac{2 n}{n+1}\right) f f^{\prime \prime}-\left(-f^{\prime}\right)^{2}+g f^{\prime \prime}-K f^{\prime}-M^{2} f^{\prime}=0 \\
& A g^{\prime \prime \prime}+\left(-f^{\prime \prime}\right)^{n-1} g^{\prime \prime \prime}-(n-1)\left(-f^{\prime \prime}\right)^{n-2} f^{\prime \prime \prime} g^{\prime \prime}+\left(\frac{2 n}{n+1}\right) f g^{\prime \prime}-\left(-g^{\prime}\right)^{2}+g g^{\prime \prime}-K g^{\prime}-M^{2} g^{\prime}=0 \\
& {\left[\left\{1+R\left(1+\left(\theta_{w}-1\right) \theta\right)^{3}\right\} \theta^{\prime}\right]^{\prime}+\operatorname{Pr}\left(\frac{2 n}{n+1}\right) f \theta^{\prime}+\operatorname{Pr} g \theta^{\prime}=0} \\
& \phi^{\prime \prime}+S c\left(\frac{2 n}{n+1}\right) f \phi^{\prime}+S c g \phi^{\prime}-S c k_{1} \phi(1-\phi)^{2}=0, \\
& \begin{array}{l}
f=0, g=0, f^{\prime}=1, g^{\prime}=\alpha, \theta^{\prime}=-\gamma(1-\theta), \phi^{\prime}(0)=k_{2} \phi(0) \text { at } \eta=0 \\
f^{\prime} \rightarrow 0, g^{\prime} \rightarrow 0, \theta \rightarrow 0, \phi(\infty) \rightarrow 1 \text { when } \eta \rightarrow \infty
\end{array}
\end{aligned}
$$

Here skin friction coefficient along $x, y$ directions and local Nusselt number, which are important engineering design quantities in materials processing, take the form:

$$
\begin{aligned}
& C f_{x}=\frac{A f^{\prime \prime}(0)-\left(-f^{\prime \prime}(0)\right)^{n}}{\operatorname{Re}_{b}^{1 / n+1}}, \\
& C f_{y}=\frac{\frac{V_{w}}{U_{w}}\left[A g^{\prime \prime}(0)+\left(-f^{\prime \prime}(0)\right)^{n-1} g^{\prime \prime}(0)\right]}{\operatorname{Re}_{b}^{1 / n+1}}, \\
& N u_{x}=-\frac{\left[1+R\left\{1+\left(\theta_{w}-1\right) \theta(0)\right\}^{3}\right]}{\operatorname{Re}_{b}^{-\frac{1}{n+1}}} \theta^{\prime}(0)
\end{aligned}
$$

It is noted that the dimensionless mass flux denoted by Sherwood number $S h_{x}$ is now identically zero.

\section{ADM SOLUTION}

The system of coupled ordinary differential equations (21)-(24) with associated boundary conditions $(25)$ constitute a $10^{\text {th }}$ order, nonlinear, multi-degree boundary value problem. Numerous methods are available to solve such systems including shooting methods, finite element methods, homotopy methods etc. Here a semi-analytical/numerical method called the Adomain Decomposition Method (ADM) is employed. In ADM, the analytic approximate solutions to a nonlinear equation are obtained without linearization and discretization yielding more accurate results. Therefore, ADM has been deployed recently in many sophisticated 
multi-physical fluid dynamic problems including magnetic bio-lubrication [38], hydromagnetic cilia propulsion [39] and swirling bioconvection nanofluid flows [40]. Adomain [31] deployed an infinite series solution for the unknown functions, utilized recursive relations and also produced an alternative approach for polynomial expansions to achieve faster convergence. Applying the standard procedure of ADM with standard notation i.e.,

$L u(y)+R u(y)+N u(y)=Q(y)$

which is derived from the form $D\{u)\}=Q(y)$. Here $D$ signifies differential operator of linear term $\{L+R\}$ with $L$ being the highest ordered derivative which is easily invertible and $R$ is the remaining linear part. The nonlinear part is $N u(y)$. It follows that the standard form can be written as follows:

$u(y)=L^{-1}\{Q(y)\}-L^{-1}\{R u(y)\}-L^{-1}\{N u(y)\}$

Next we introduce $L_{1}=\frac{d^{3}}{d \eta^{3}}()$ and $L_{2}=\frac{d^{2}}{d \eta^{2}}()$ with invertible operators $L_{1}^{-1}()=\int_{0}^{\eta} \int_{0}^{\eta} \int_{0}^{\eta}() d \eta d \eta d \eta$ and $L_{2}^{-1}()=\int_{0}^{\eta} \int_{0}^{\eta}() d \eta d \eta$. Thus, arranging all the Eqns. (21)-(24) and by writing them as infinite series using recursive relations with initial guesses, the approximate analytical solutions can be obtained for the variables (primary velocity, $f^{\prime}$, secondary velocity $g^{\prime}$, temperature $\theta$, concentration $\phi$ ). The ADM power series expansions are listed in the Appendix.

\section{RESULTS AND DISCUSSION}

The influence of selected parameters on primary and secondary velocity components, temperature and concentration distributions is visualized in Figs. 2-17. Moreover, computed values of the primary and secondary skin friction $\left(-f^{\prime \prime}(0),-g^{\prime \prime}(0)\right)$ and Nusselt number $\left.-\theta^{\prime}(0)\right)$ are displayed in Table 2. Validation of the ADM solutions is provided for the primary and secondary skin friction coefficients with different stretching sheet ratio parameter $(\alpha)$ in Table 1 for comparison with the ND Solve shooting quadrature Mathematica solutions of Hayat et al. [30] $A=M=K=R=0$ i.e. in the absence of Sisko non-Newtonian, magnetic, porous medium and radiative effects. Reasonable agreement is achieved and confidence in the present ADM solutions is therefore high. 
Table 1: Comparison of skin friction coefficients for different values of $\alpha$ when $A=M=K=R$

\begin{tabular}{|c|c|c|c|c|}
\hline$\alpha$ & $-f^{\prime \prime}(0)$ & $-f^{\prime \prime}(0)$ & $-g^{\prime \prime}(0)$ & $-g^{\prime \prime}(0)$ \\
& Hayat et al.,$[30]$ & ADM results & Hayat et al., [30] & ADM results \\
\hline 0.3 & 1.055234 & 0.9475 & 0.234632 & 0.2500 \\
\hline 0.5 & 1.090504 & 0.9828 & 0.458035 & 0.4527 \\
\hline 0.8 & 1.149069 & 1.0413 & 0.859627 & 1.1684 \\
\hline 1.0 & 1.172897 & 1.0651 & 1.698852 & 1.6936 \\
\hline
\end{tabular}

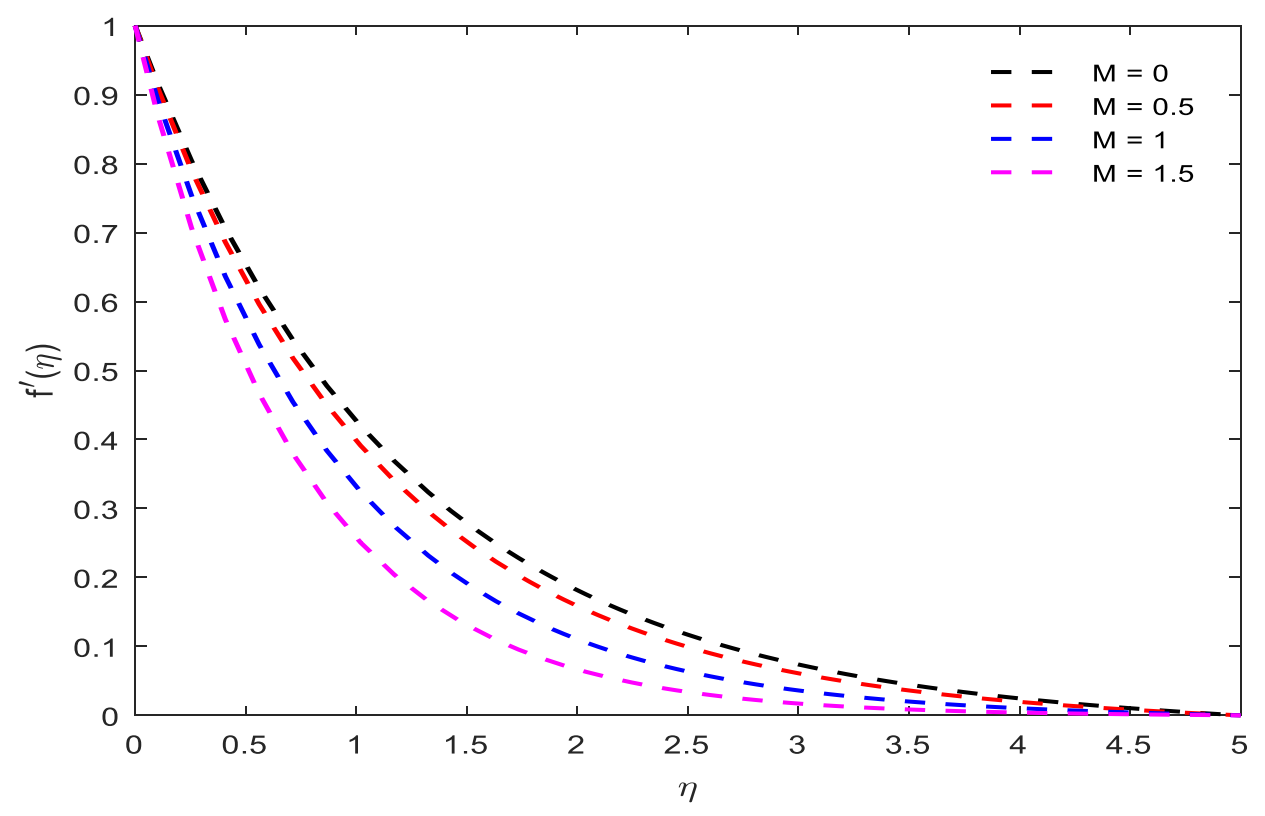

Figure 2: Influence of magnetic parameter on velocity component in x-direction for $\gamma=0.3, K=0.5, A=\alpha=n=S c=1.0, \operatorname{Pr}=4.0, R=0.3, k_{1}=k_{2}=0.5$

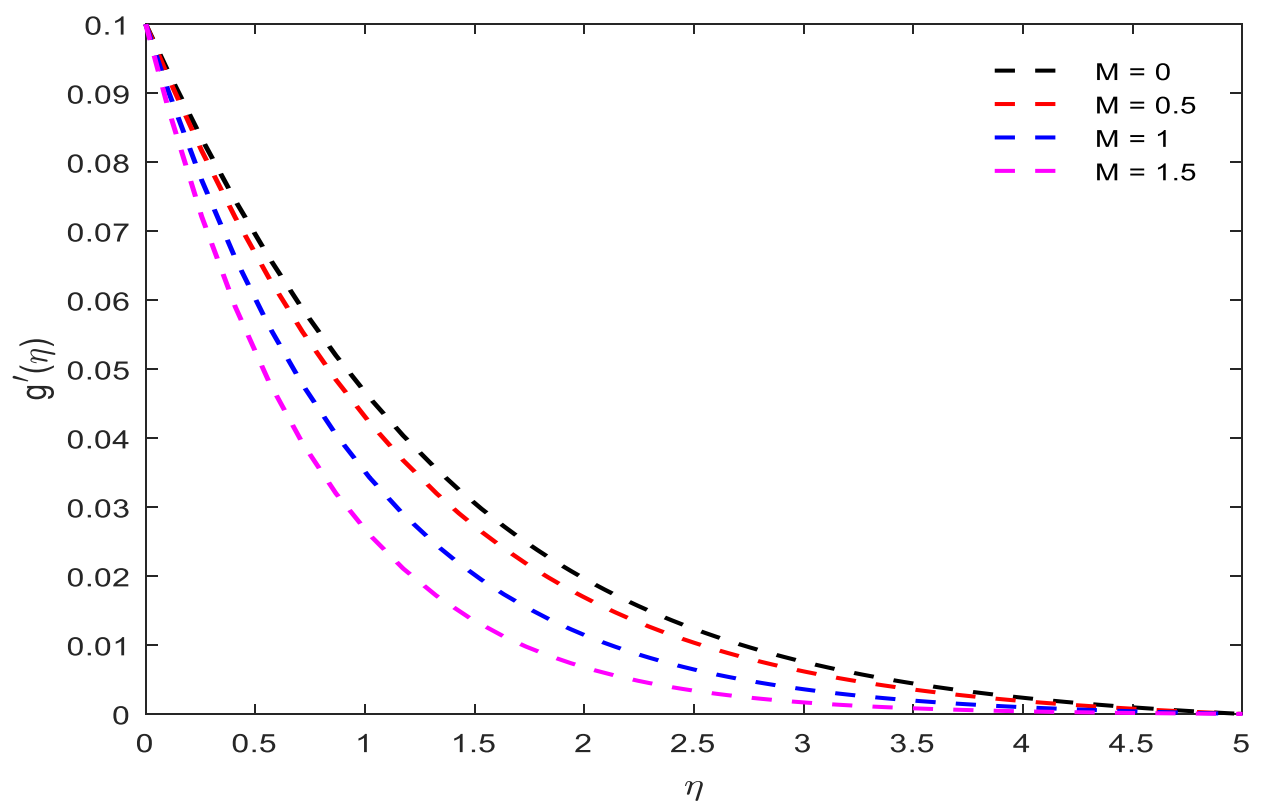

Figure 3: Influence of magnetic parameter on velocity component in y-direction for $\gamma=0.3, K=0.5, A=\alpha=n=S c=1.0, R=0.3, \operatorname{Pr}=4.0, k_{1}=k_{2}=0.5$ 


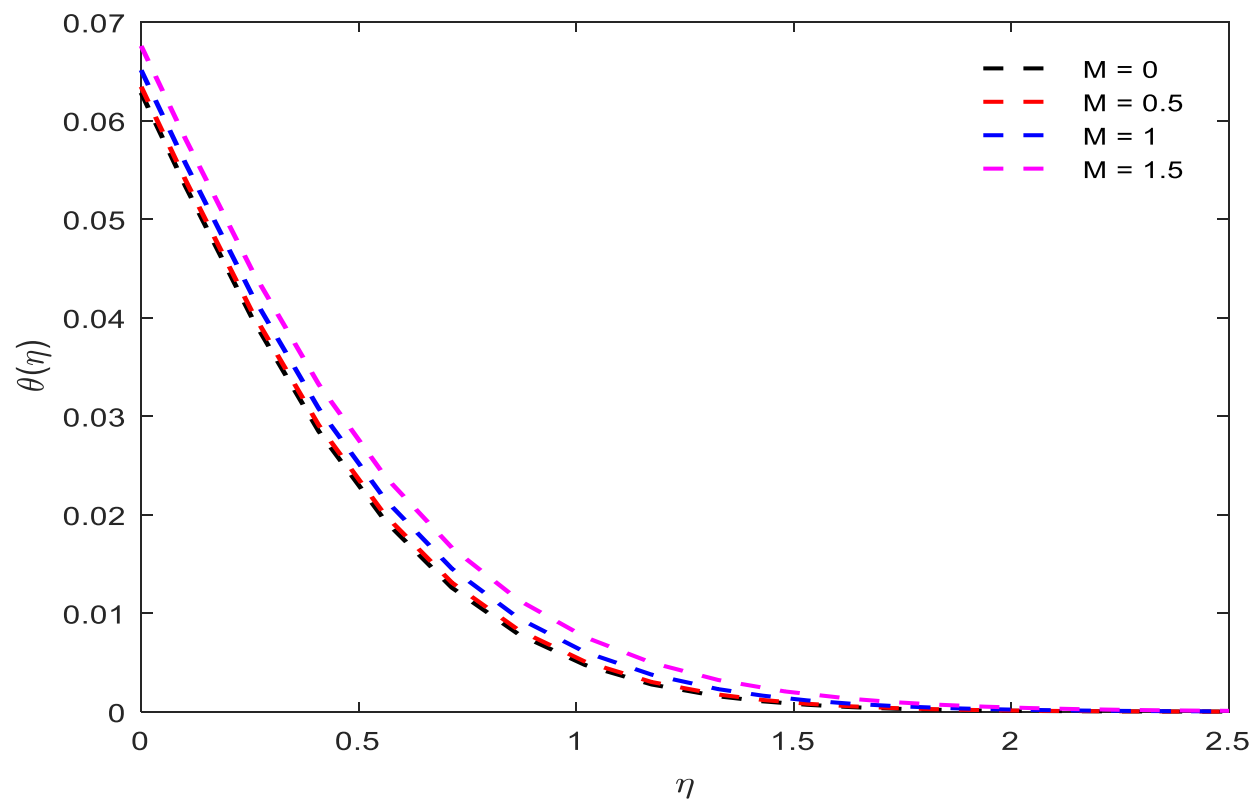

Figure 4: Influence of magnetic parameter on temperature for $\gamma=0.3, K=0.5, A=\alpha=n=S c=1.0, R=0.3, \operatorname{Pr}=4.0, k_{1}=k_{2}=0.5$

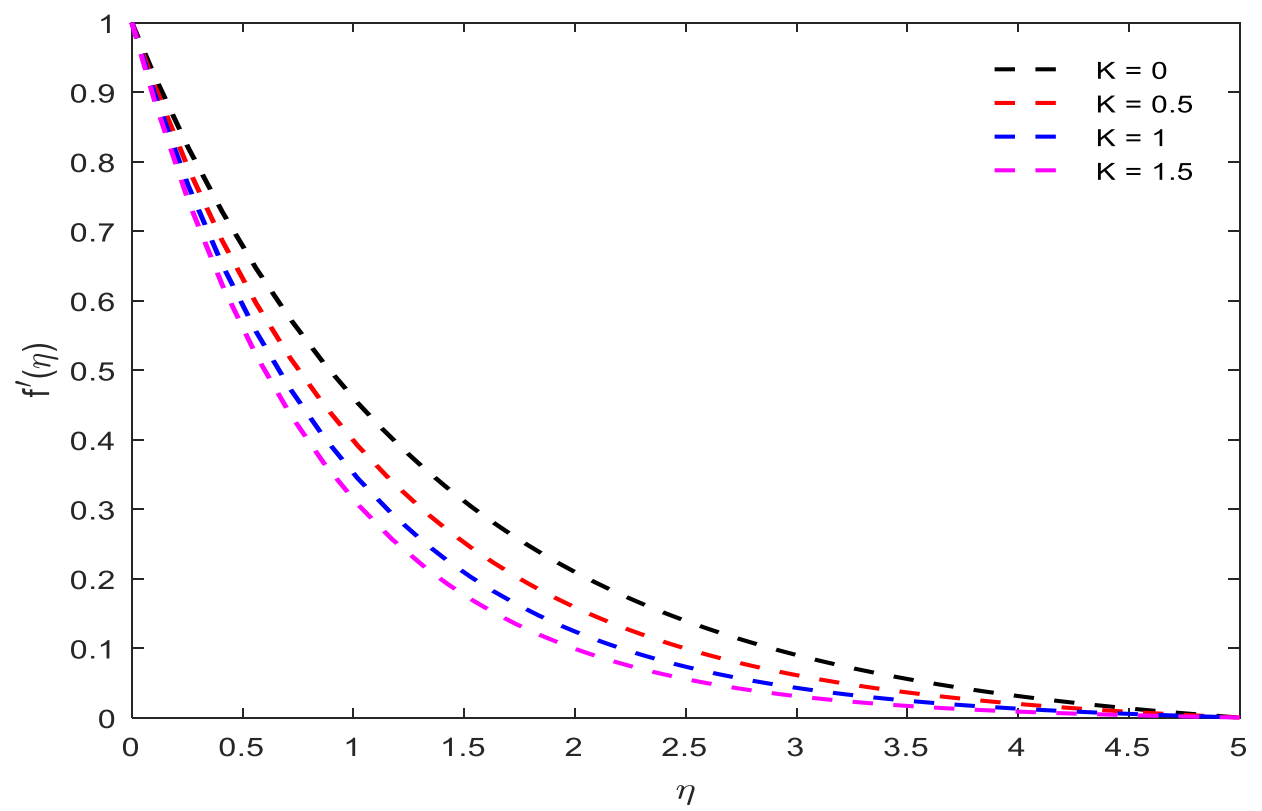

Figure 5: Influence of permeability parameter on velocity component in x-direction for $\gamma=0.3, M=0.3, A=\alpha=n=S c=1.0, R=0.3, \operatorname{Pr}=4.0, k_{1}=k_{2}=0.5$ 


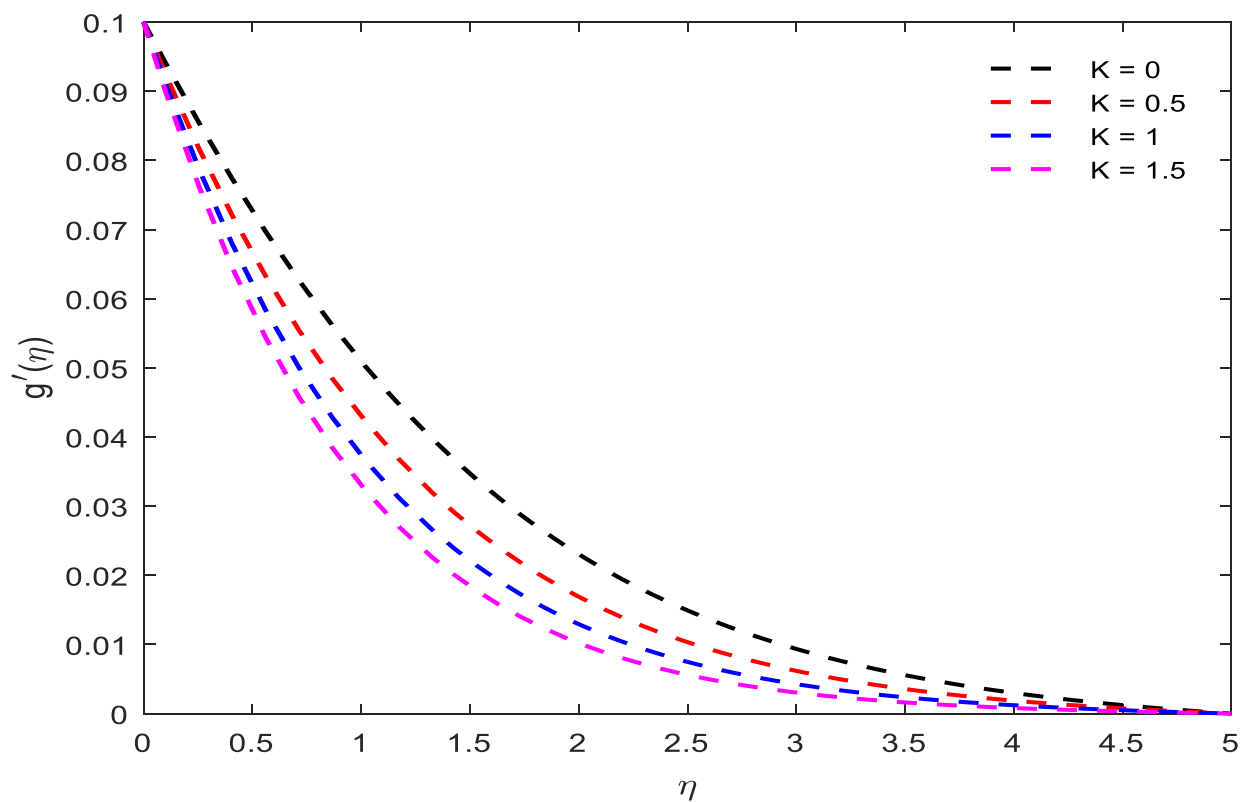

Figure 6: Influence of permeability parameter on velocity component in y-direction for $\gamma=0.3, M=0.3, A=\alpha=n=S c=1.0, R=0.3, \operatorname{Pr}=4.0, k_{1}=k_{2}=0.5$

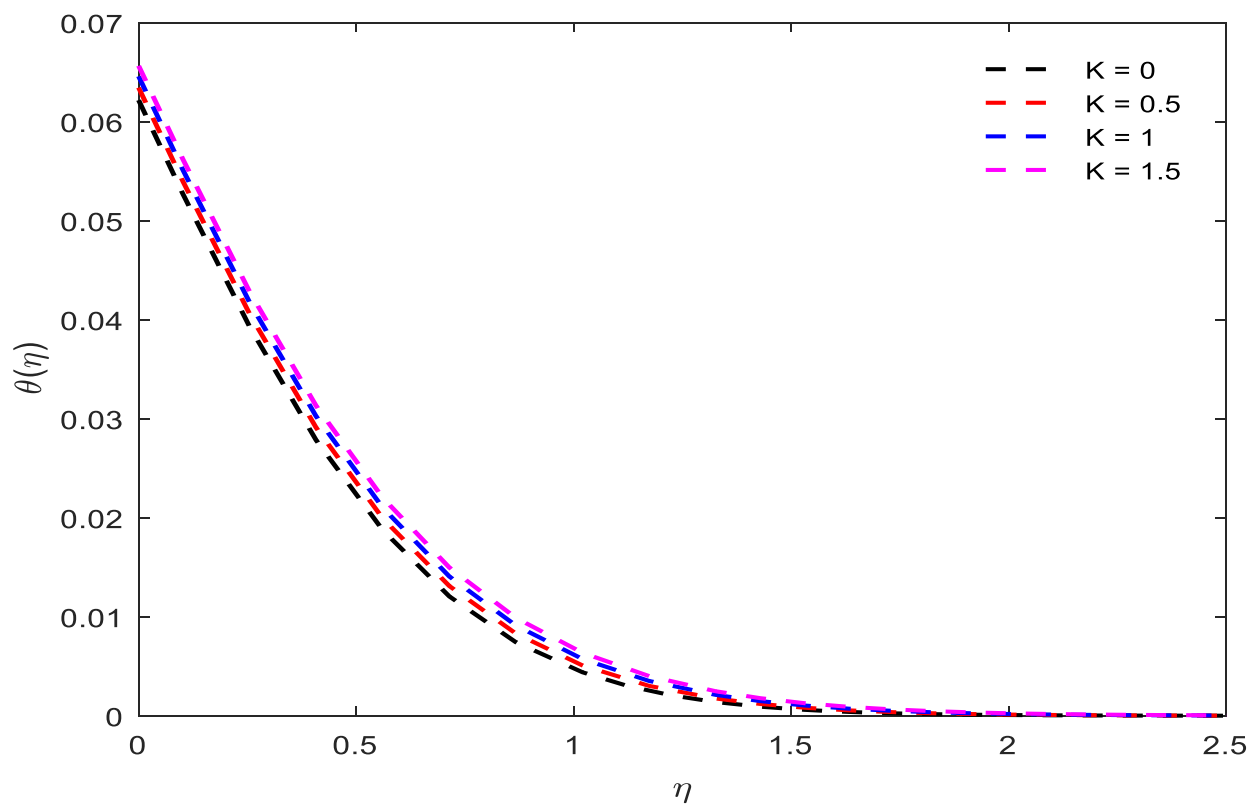

Figure 7: Influence of permeability parameter on temperature for $\gamma=0.3, M=0.3, A=\alpha=n=S c=1.0, R=0.3, \operatorname{Pr}=4.0, k_{1}=k_{2}=0.5$ 


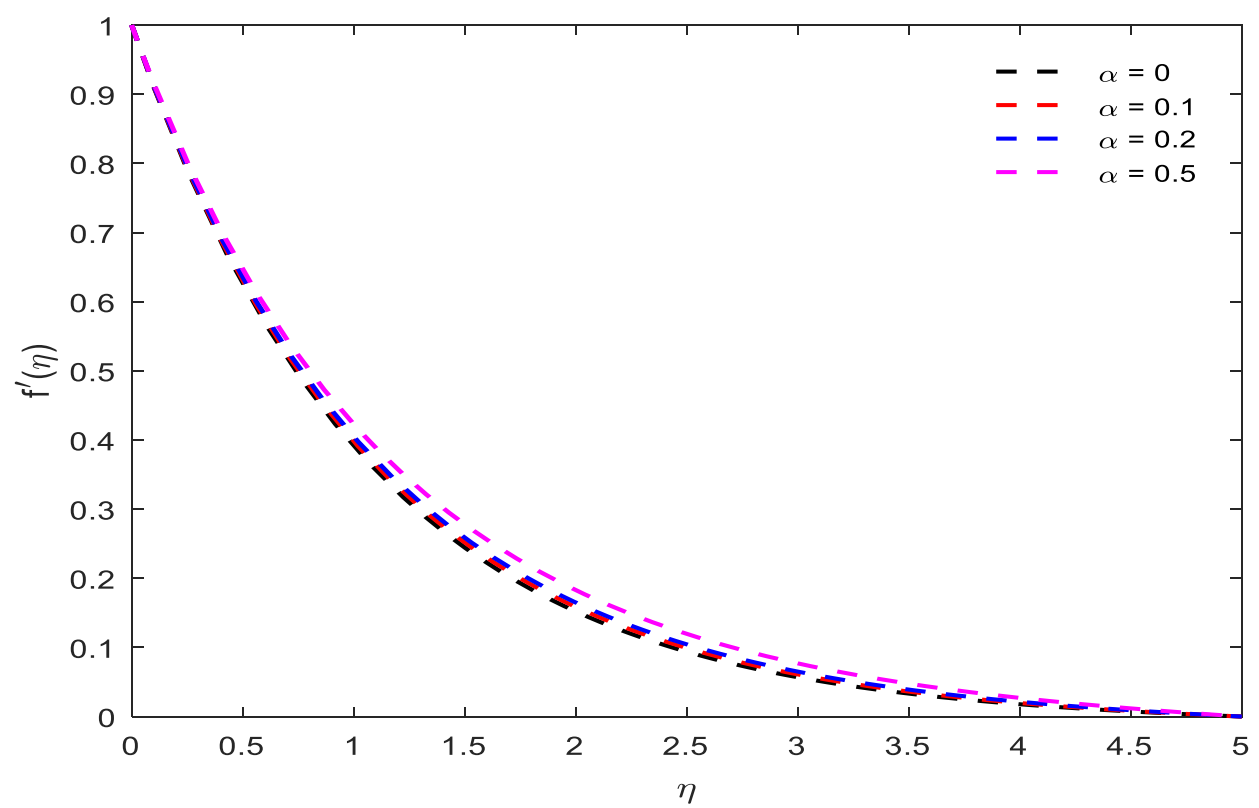

Figure 8: Influence of ratio parameter on velocity component in $\mathrm{x}$-direction for $\gamma=0.3, M=0.3, K=0.5, A=n=S c=1.0, R=0.3, \operatorname{Pr}=4.0, k_{1}=k_{2}=0.5$

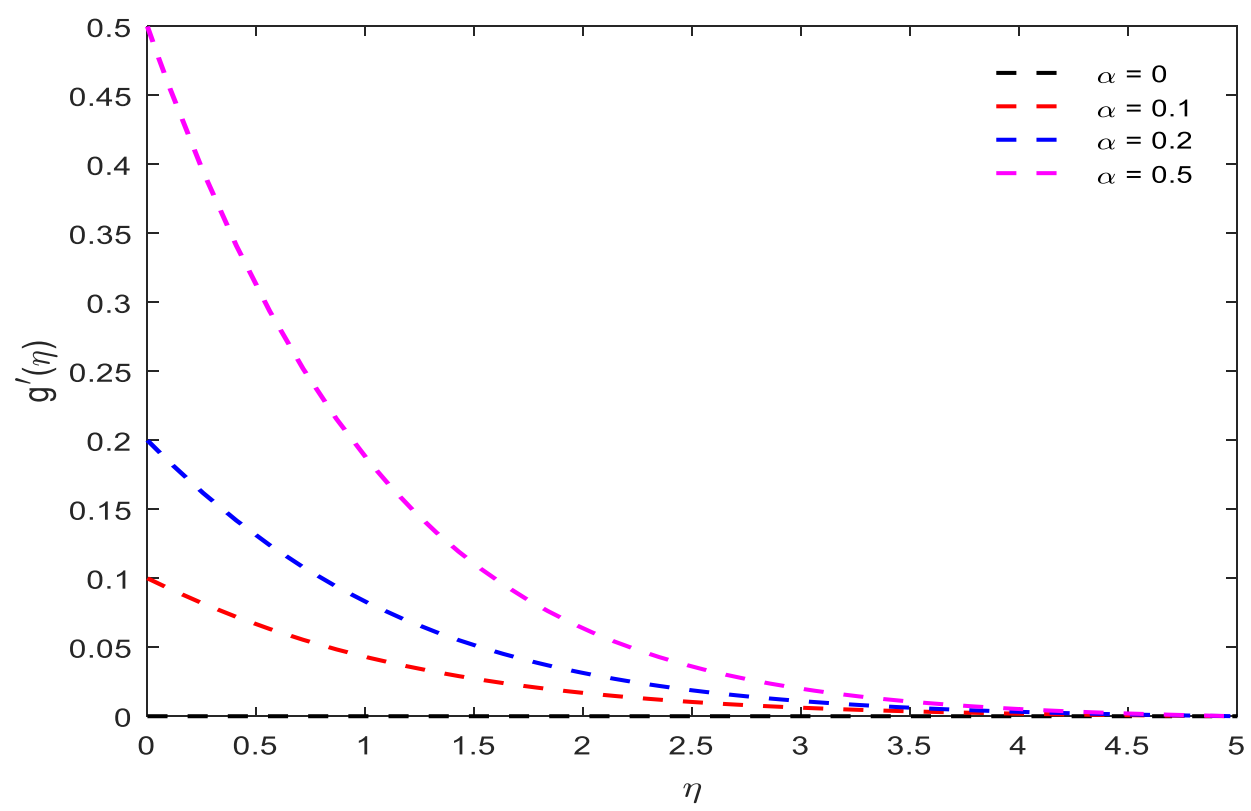

Figure 9: Influence of ratio parameter on velocity component in y-direction for $\gamma=0.3, M=0.3, K=0.5, A=n=S c=1.0, R=0.3, \operatorname{Pr}=4.0, k_{1}=k_{2}=0.5$ 


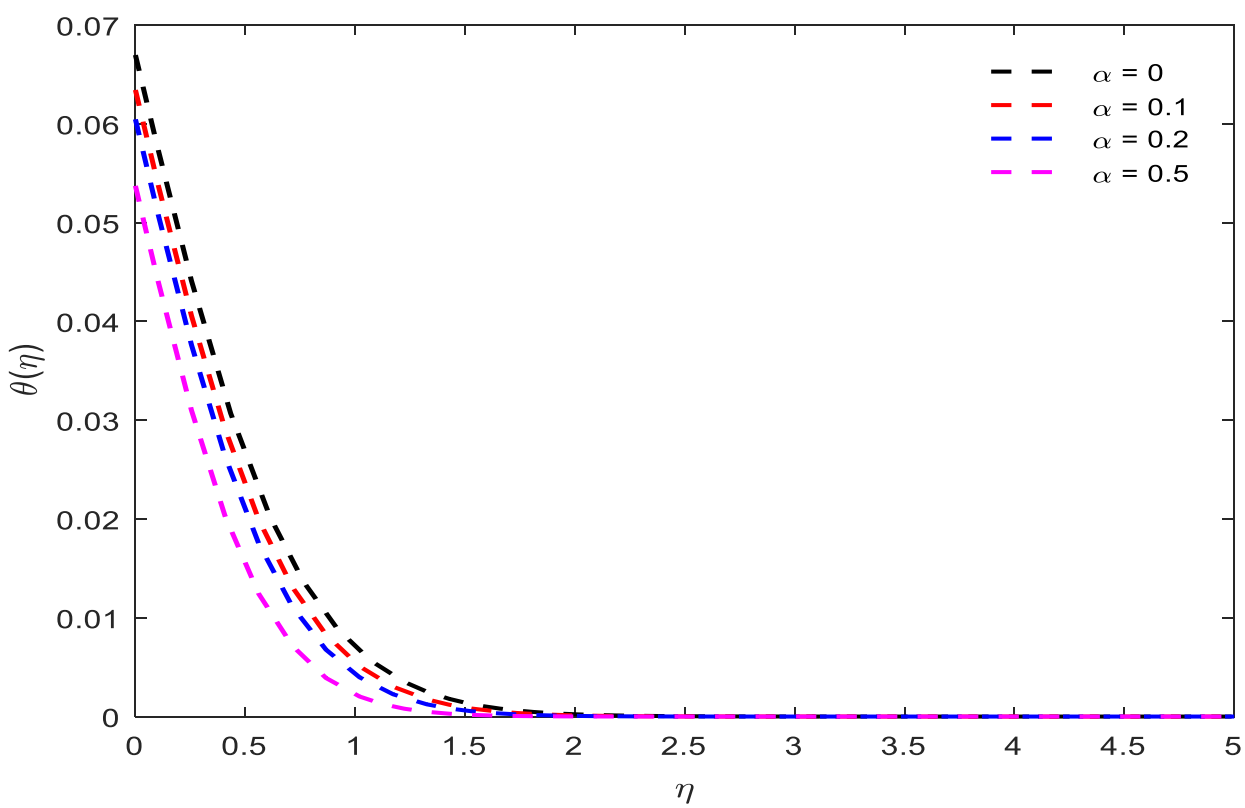

Figure 10: Influence of ratio parameter on temperature for $\gamma=0.3, M=0.3, K=0.5, A=n=S c=1.0, R=0.3, \operatorname{Pr}=4.0, k_{1}=k_{2}=0.5$

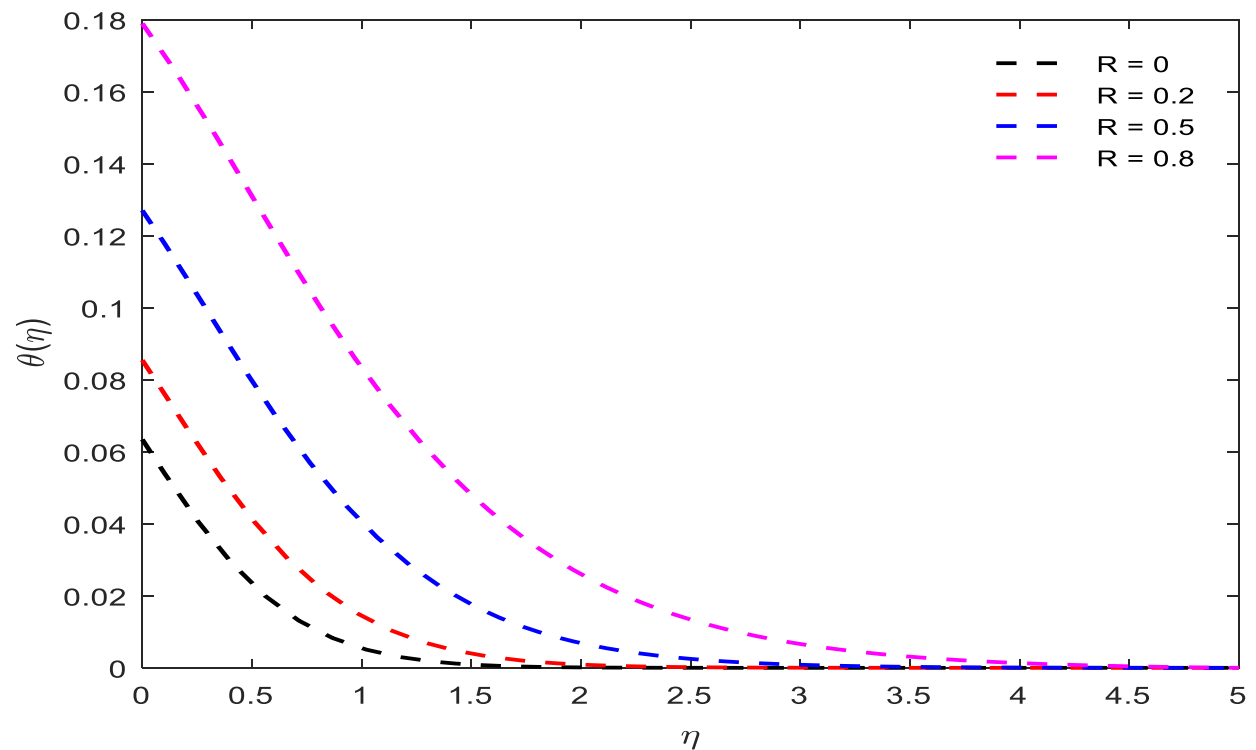

Figure 11: Influence of thermal radiation parameter on temperature for $\gamma=0.3, M=0.3, K=0.5, A=\alpha=n=S c=1.0, \operatorname{Pr}=4.0, k_{1}=k_{2}=0.5$ 


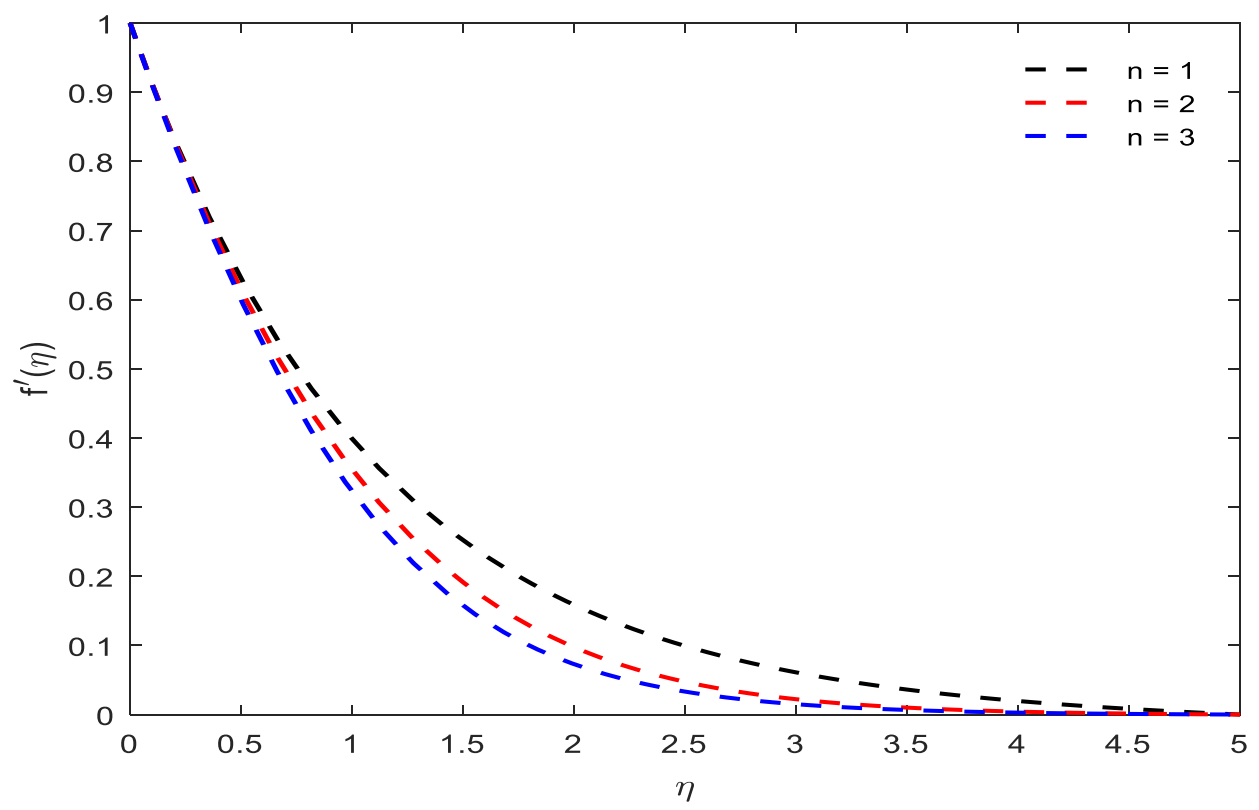

Figure 12: Influence of material parameter on velocity component in $\mathrm{x}$-direction for $\gamma=0.3, M=0.3, K=0.5, A=\alpha=S c=1.0, R=0.3, \operatorname{Pr}=4.0, k_{1}=k_{2}=0.5$

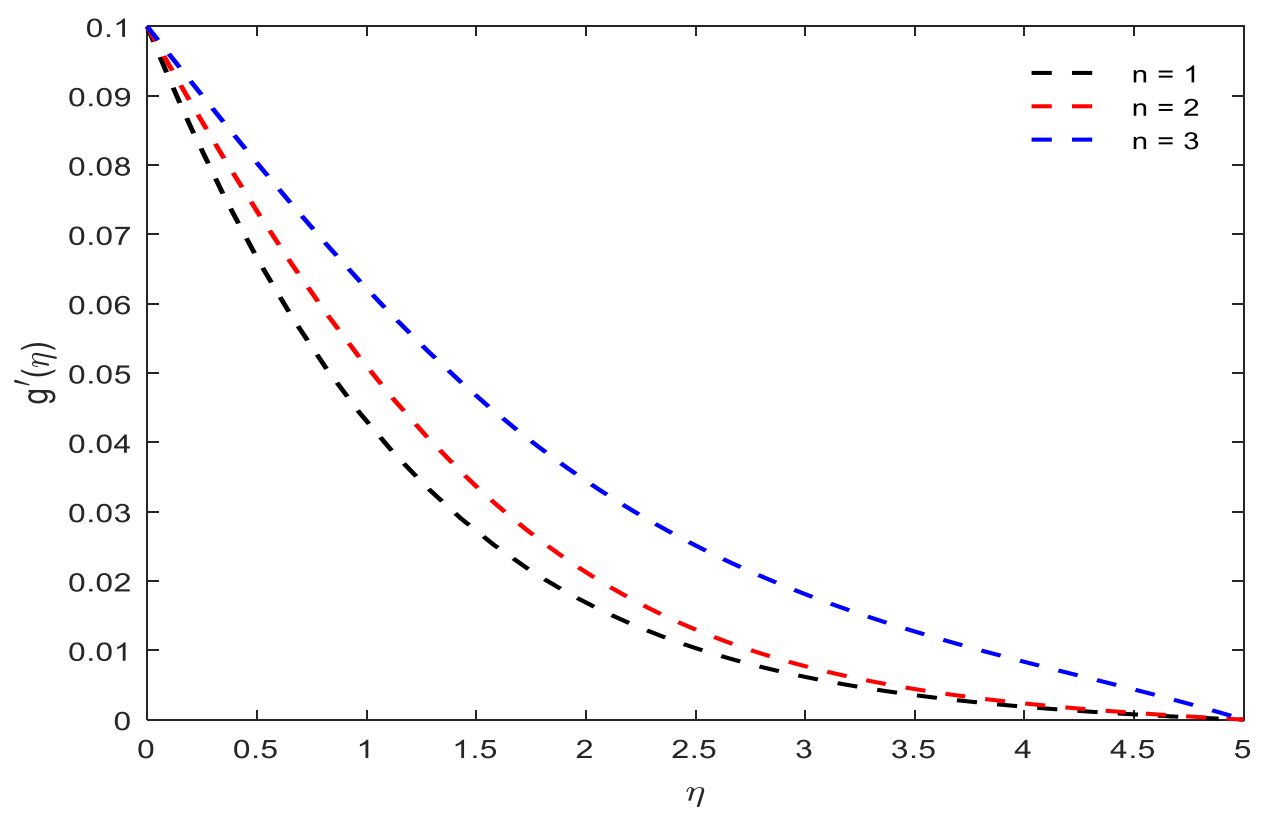

Figure 13: Influence of material parameter on velocity component in y-direction for $\gamma=0.3, M=0.3, K=0.5, A=\alpha=S c=1.0, R=0.3, \operatorname{Pr}=4.0, k_{1}=k_{2}=0.5$ 


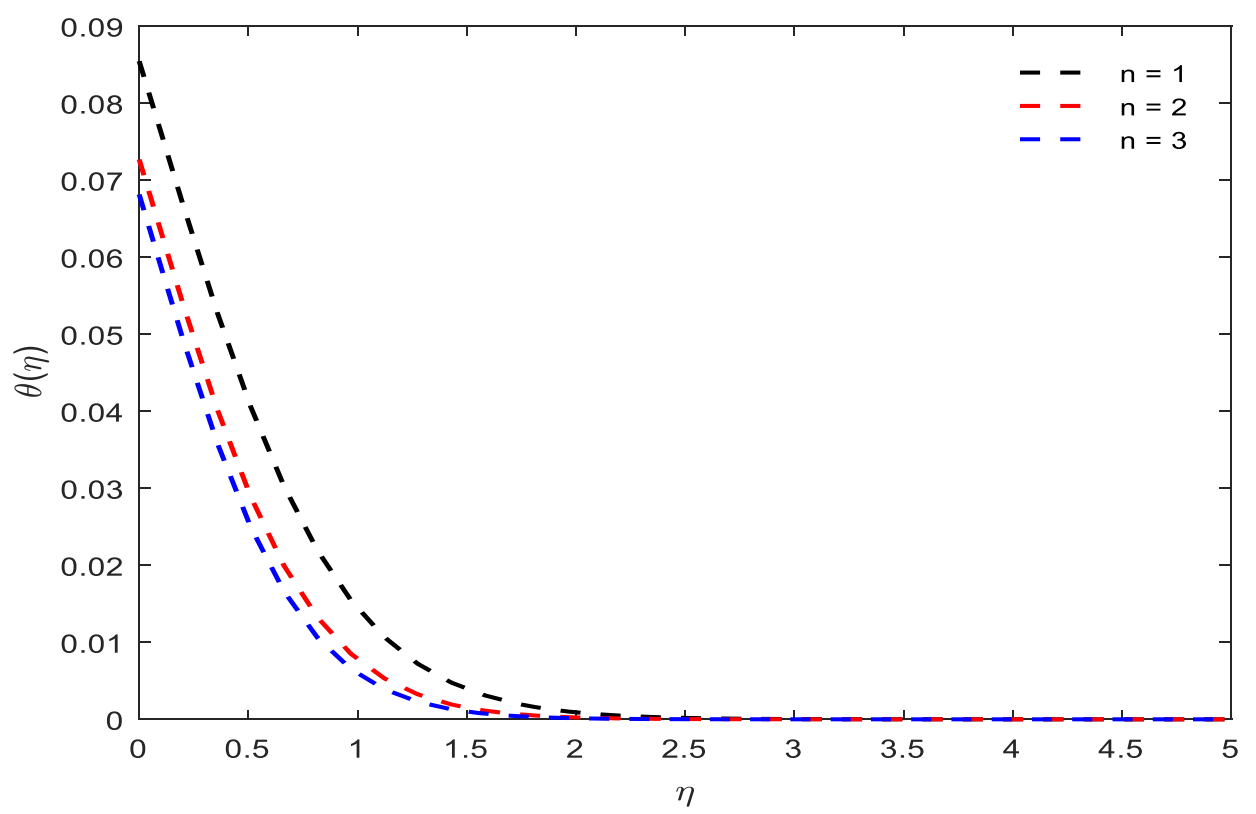

Figure 14: Influence of material parameter on temperature for $\gamma=0.3, M=0.3, K=0.5, A=\alpha=S c=1.0, R=0.3, \operatorname{Pr}=4.0, k_{1}=k_{2}=0.5$

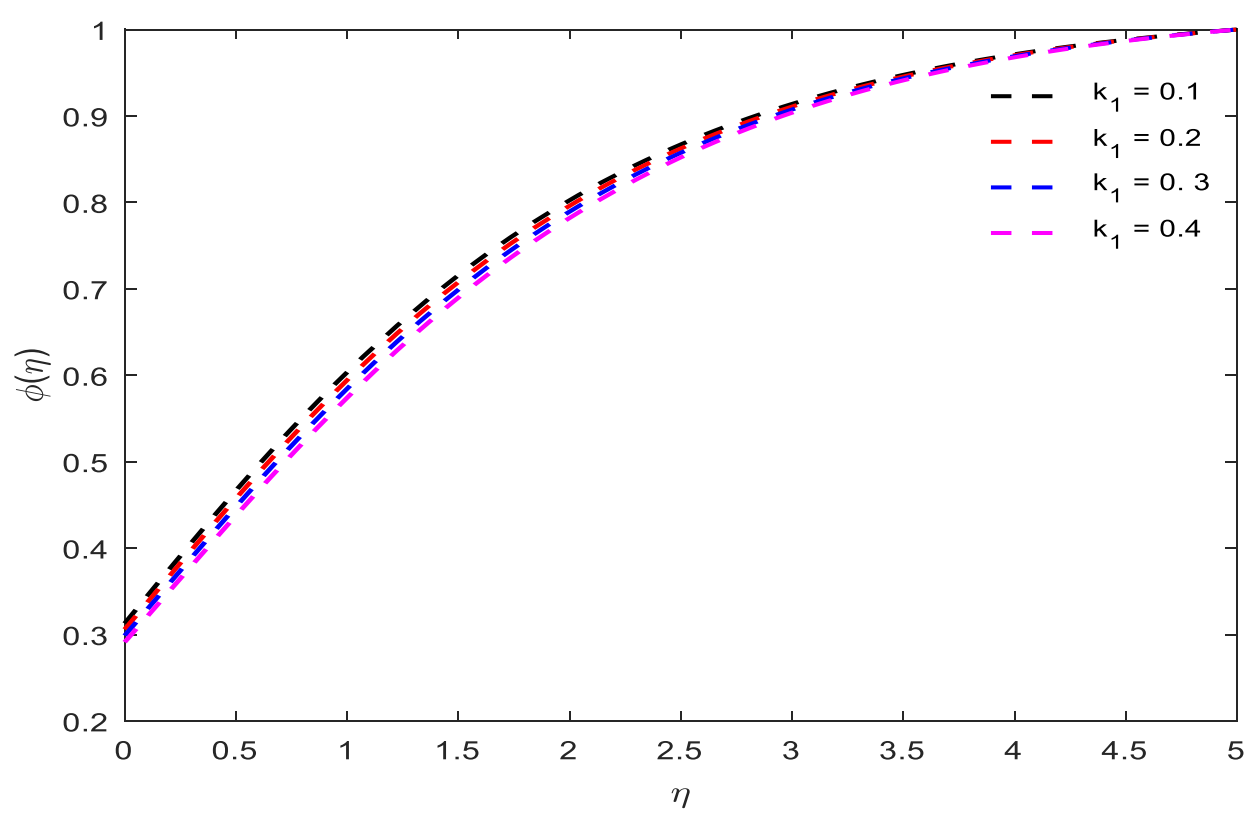

Figure 15: Influence of strength of homogenous chemical reaction parameter on concentration for $\gamma=0.3, M=0.3, K=0.5, A=\alpha=n=S c=1.0, R=0.3, \operatorname{Pr}=4.0, k_{2}=0.5$ 


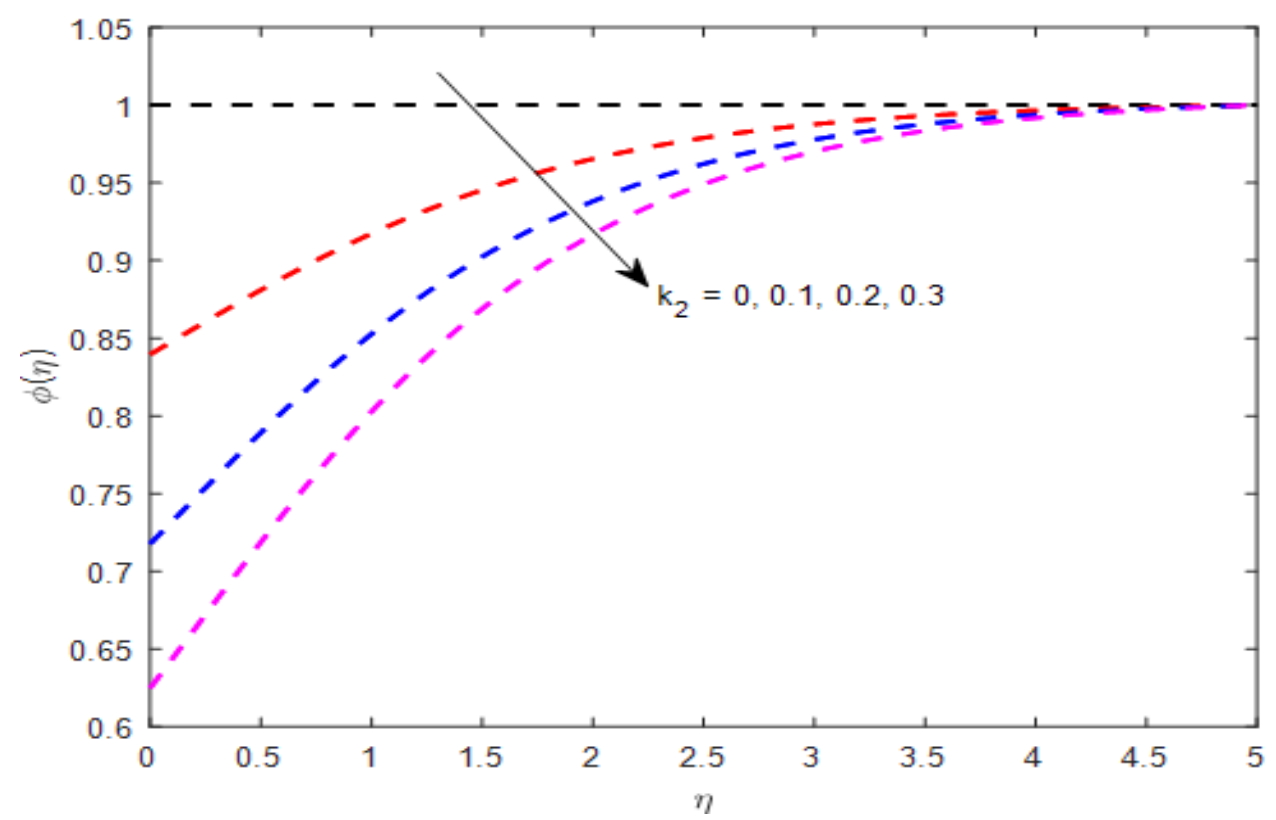

Figure 16: Influence of strength of heterogenous chemical reaction parameter on concentration for $\gamma=0.3, M=0.3, K=0.5, A=\alpha=n=S c=1.0, R=0.3, \operatorname{Pr}=4.0, k_{1}=0.5$

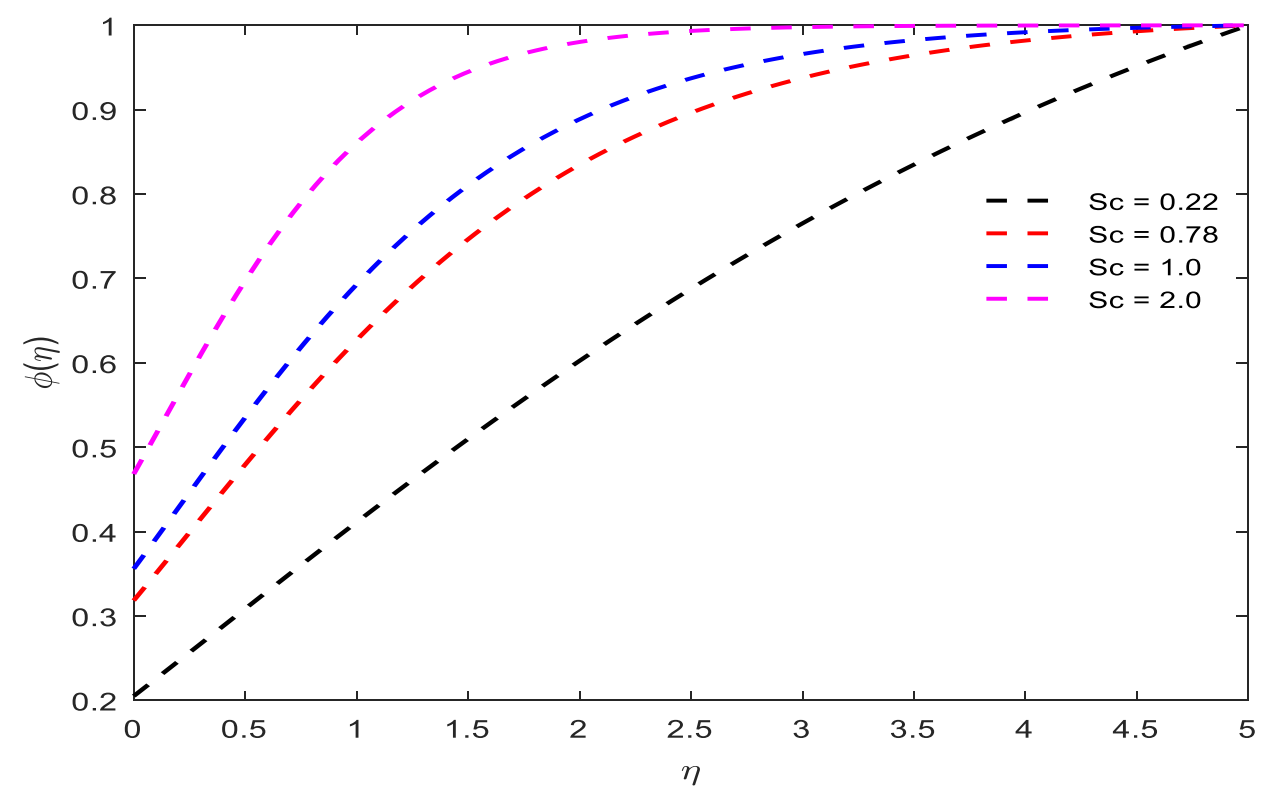

Figure 17: Influence of Schmidt number on concentration for $\gamma=0.3, M=0.3, K=0.5, A=\alpha=n=1.0, R=0.3, \operatorname{Pr}=4.0, k_{1}=k_{2}=0.5$ 
Table 2: Values of $-f^{\prime \prime}(0),-g^{\prime \prime}(0)$ and $-\theta^{\prime}(0)$ for different values of $A, M, K, \alpha, n$ and $R$

\begin{tabular}{|c|c|c|c|c|c|c|c|c|}
\hline$A$ & $M$ & $K$ & $\alpha$ & $n$ & $R$ & $-f^{\prime \prime}(0)$ & $-g^{\prime \prime}(0)$ & $-\theta^{\prime}(0)$ \\
\hline 0.0 & 0.0 & 0.0 & 0.3 & 1.0 & 0.2 & 0.9475 & 0.25 & 0.0923 \\
\hline 0.5 & & & & & & 0.7761 & 0.2045 & 0.0925 \\
\hline 1.0 & & & & & & 0.6751 & 0.1778 & 0.0926 \\
\hline & 0.5 & & & & & 0.7597 & 0.2049 & 0.0925 \\
\hline & 1.0 & & & & & 0.9743 & 0.2731 & 0.0923 \\
\hline & 1.5 & & & & & 1.2546 & 0.361 & 0.0918 \\
\hline & & 0.5 & & & & 0.8369 & 0.2295 & 0.0924 \\
\hline & & 1.0 & & & & 0.9743 & 0.2731 & 0.0923 \\
\hline & & 1.5 & & & & 1.095 & 0.3111 & 0.0921 \\
\hline & & & 0.1 & & & 0.699 & 0.049 & 0.0918 \\
\hline & & & 0.2 & & & 0.6751 & 0.1778 & 0.0926 \\
\hline & & & 0.5 & & & 0.654 & 0.3391 & 0.0932 \\
\hline & & & & 1.0 & & 0.6751 & 0.1778 & 0.0926 \\
\hline & & & & 1.5 & & 0.9914 & 0.0599 & 0.1275 \\
\hline & & & & 2.0 & & 0.7245 & 0.1211 & 0.0936 \\
\hline & & & & & 1.0 & 0.7597 & 0.2049 & 0.0826 \\
\hline & & & & & 2.0 & 0.7597 & 0.2049 & 0.0671 \\
\hline & & & & & 3.0 & 0.7597 & 0.2049 & 0.0551 \\
\hline
\end{tabular}

In the model Eqns. (21)-(24) with boundary conditions (25), 12 parameters arise and the default values set in the ADM computations are as follows: $A$ (Sisko material parameter) $=$ 1.0, $n$ (Sisko power-law index $)=1.0, K$ (permeability parameter $)=1.0, M$ (magnetic parameter $)=1.0, \alpha$ (bidirectional sheet stretching ratio $)=1.0, \theta_{w}($ temperature ratio parameter )$=0.1, k_{1}$ (homogenous reaction strength $)=0.5, k_{2}$ (heterogeneous reaction strength $)=0.5, \operatorname{Pr}$ $($ Prandtl number $)=4.0, R($ radiative parameter $)=1.0, S c($ Schmidt number $)=1.0, \gamma($ thermal Biot number $)=0.3$.

Figs. 2-4 illustrate the influence of magnetic body force parameter on the primary (x-direction) and secondary ( $y$-direction) velocity components and temperature. There is a significant reduction in the primary velocity (velocity component in $x$-direction) with greater magnetic field, as observed in Fig. 2. The Lorentz body force associated with the vertical magnetic field has components in both the $x$ - and $y$-directions, which appear as the terms $-M^{2} f^{\prime}$ and $-M^{2} g^{\prime}$ in eqns. (21) and (22), respectively. This is a retarding force and impedes the motion of the sheet. Clearly maximum primary velocity is achieved in the absence of a magnetic field i.e. $M=0$ (electrically non-conducting case) and the minimum primary velocity corresponds to the strongest magnetic field case of $M=1.5$. Fig. 3 demonstrates that while a similar reduction in secondary velocity is induced with increasing magnetic parameter, the magnitudes of the 
secondary velocity are significantly lower than the primary velocity. In both Figs 2 and 3 asymptotically smooth profiles in velocity components from the sheet surface (wall) to the edge of the boundary layer (free stream) are observed indicating that a sufficiently high infinity boundary condition has been prescribed in the ADM computations. In fig. 3 the increase in magnetic parameter is seen to enhance temperatures throughout the flow domain. Although magnetic terms do not arise in the energy conservation Eqn. (23), the coupling of the temperature $(\theta)$ Eqn. with the primary and secondary momentum equations via for example the terms, $\operatorname{Pr}\left(\frac{2 n}{n+1}\right) f \theta^{\prime}, \operatorname{Pr} g \theta^{\prime}$ implies that there is an indirect influence of the magnetic field on the temperature. The supplementary work done in dragging the Sisko fluid against the action of the transverse magnetic field is expended as heat i.e. thermal energy. This energizes the Sisko fluid and leads to an elevation in temperatures with greater magnetic parameter, $M$. Clearly the regime is cooled in the absence of a magnetic field and heated substantially with strong vertical magnetic field strength. This also concurs with the findings of Hayat et al. [30].

Figs. 5-7 illustrate the impact of the inverse permeability parameter, $K$, on primary (xdirection) and secondary ( $y$-direction) velocity components and temperature. As in the case of the magnetic body force, the impedance due to the porous medium also results in a Darcian drag force component in both primary and secondary momentum equations. These components are linear in nature i.e. $-K f^{\prime}$ and $-K g^{\prime}$ clearly resistive. The parameter $K$ is infact inversely related to the actual permeability of the porous medium, $k$, as defined in Eqn. (17). As $K$ is increased, $k$ is decreased implying that there is greater solid fiber resistance to the percolating flow since the permeability is lower. This manifests in an increase in Darcian drag force components in both primary and secondary momentum equations and leads to significant deceleration in both primary and secondary flows, as observed in Figs. 5 and 6. Conversely with increasing $K$ parameter (decreasing permeability, $k$, of the medium) there is a boost in temperatures, as observed in Fig. 7. This is attributable to the enhanced thermal conduction present in the porous medium with greater concentration of solid fibers at lower permeabilities. Evidently therefore cooling of the regime is achieved with purely fluid regimes i.e. the vanishing porous medium case $(K=0$ implying $k \rightarrow \infty)$.

Figs. 8-10 depict the variations in primary ( $x$-direction) and secondary ( $y$-direction) velocity components and temperature with sheet stretching ratio $(\alpha=d / c)$. When $\alpha=0$ the sheet is stagnant i.e. no stretching occurs and two-dimensional flow is achieved. For $\alpha=1$, 
axisymmetric stretching is produced i.e. equal stretching rates in both $\mathrm{x}$ and $\mathrm{y}$ directions. $\mathrm{A}$ weak acceleration is induced in the primary flow with increasing sheet stretching ratio, $\alpha$ (fig. 8); a much stronger enhancement in secondary velocity is induced in Fig. 9, and the effect is greater at the wall and progressively diminishes into the free stream. Again, asymptotically smooth profiles are achieved in both Figs. 8 and 9 in the free stream confirming the implementation of adequately large infinity boundary conditions in the ADM solution. A notable depletion in temperature accompanies a rise in stretching ratio parameter (Fig. 10) and again this is largely concentrated in the near-wall region.

Fig. 11 illustrates the distribution in temperature with various radiation parameter $(R)$ values. This parameter feature sin the augmented diffusion term in the energy conservation Eqn. (23) i.e. $\left[\left\{1+R\left(1+\left(\theta_{w}-1\right) \theta\right)^{3}\right\} \theta^{\prime}\right]^{\prime} . \mathrm{R}=\frac{16 \sigma^{*}}{3 k k^{*}} T \infty^{3}$ and embodies the relative contribution of thermal radiation heat transfer to thermal conduction heat transfer. For $R=0$ radiative flux vanishes. As $R$ increases the Sisko fluid regime is progressively energized via radiation and temperatures are strongly boosted. The most dramatic impact is nearer the wall although the temperature elevation is sustained some distance from the wall. Evidently in mathematical models of high-temperature materials processing of magnetic polymers, the neglection of radiative heat transfer will significantly under-predict temperatures. It is important to include radiative effects albeit with simple algebraic flux models and in this regard the Rosseland model does capture the modification in temperatures quite reasonably. Similar observations have been made in Lu et al. [26] and Bég et al. [29].

Figs. 12-14 depict the evolution in primary ( $x$-direction) and secondary ( $y$-direction) velocity components and temperature with Sisko rheological power-law index (material parameter), $n$. there is a significant reduction in primary velocity, $f^{\prime}(\eta)$, with greater $n$ values. Higher values of this parameter imply a significant escalation in polymer viscosity and increasingly shearshickening (dilatant) this results in a deceleration in the primary flow of the bi-directional stretching sheet (Fig. 12). Conversely the momentum lost in the primary flow is re-distributed in the secondary flow and this leads to an elevation in secondary flow velocities (Fig. 13). Secondary flow is therefore accelerated for the strong dilatant polymer whereas primary flow is decelerated. The shear-thinning case $(0<n<1)$ has been considered elsewhere [30] and is not studied here. A strong decrease in temperatures is also generated with increasing dilatant behavior $(n>1)$ as observed in Fig. 14.

Figs. 15-16 depict the impact of $k_{1}$ (homogenous reaction strength) and $k_{2}$ (heterogeneous reaction strength), respectively, on concentration distribution, $\phi(\eta)$.There is a weak decrease 
in concentration with increasing $k_{1}$ values i.e. homogenous destructive reaction depletes the concentration of the original species due to conversion to another, as observed in Fig. 15. The $k_{1}$ parameter arises in the reduced diffusion Eqn. (24), whereas $k_{2}$ (heterogeneous reaction strength) features in the concentration wall boundary condition (25) i.e. $\phi^{\prime}(0)=k_{2} \phi(0)$. A much stronger depletion in the concentration magnitudes is induced with increasing $k_{2}$ values. Maximum concentration is achieved in the absence of heterogenous reaction $\left(k_{2}=0\right)$ as seen in Fig. 16.

Fig. 17 the response in concentration $\phi(\eta)$ with Schmidt number, Sc. Schmidt number provides a quantification of the relative rate of momentum diffusion to species diffusion. When $S c=1$ both rates of diffusion are the same. $S c<1$ is representative of for example oxygen diffusion in low-weight polymers and $S c>1$ corresponds to diffusion of gases in higher density polymers. A marked enhancement in concentration magnitudes is generated with increasing Schmidt number and there is also a morphing in profiles from the approximately linear case $(S c=0.22)$ to the strongly parabolic case $(S c=2.0)$.

Table 2 shows the variation in $-f^{\prime \prime}(0)$ i.e. primary skin friction, $-g^{\prime \prime}(0)$ i.e. secondary skin friction and $-\theta^{\prime}(0)$ i.e. Nusselt number with several selected parameters i.e. $A, M, K, \alpha, n$ and $R$. Again, default values for the other parameters are as noted earlier. Primary skin friction is strongly reduced with increasing $A$ values (Sisko material parameter) whereas secondary skin friction is weakly reduced and Nusselt number is slightly increased. With increasing magnetic parameter, $M$, the primary skin friction and secondary skin friction are elevated whereas the Nusselt number is weakly reduced. An increase in inverse permeability parameter, $K$, also enhances primary and secondary skin friction whereas it reduces Nusselt number. Increasing stretching ratio parameter $(\alpha)$ weakly reduces primary skin friction but strongly elevates secondary skin friction and slightly boosts the Nusselt number at the wall. Increasing Sisko power-law index $(n)$ i.e. greater dilatant behavior is found to elevate primary skin friction and reduce secondary skin friction whereas it initially increases and thereafter reduces Nusselt number. Finally, increasing radiative parameter, $R$, exerts no tangible influence on either primary or secondary friction but strongly reduces the Nusselt number (since heat is transferred away from the wall into the body of the bi-directional stretching sheet flow leading to greater temperatures in the boundary layer). 


\section{CONCLUSIONS}

A mathematical model has been presented for steady-state, laminar, incompressible, magnetohydrodynamic flow of an electrically conducting polymeric non-Newtonian (Sisko) fluid from a bidirectional stretching sheet in a porous medium is studied theoretically. Thermal radiation flux and homogeneous-heterogeneous chemical reactions are included. Darcy's model is employed for the porous medium and Rosseland's diffusion flux model for radiation heat transfer. Convective heating conditions at the wall are also considered. The transformed dimensionless nonlinear ordinary differential equation boundary value problem with transformed boundary conditions has been solved with the semi-analytical Adomain decomposition method (ADM). Validation with earlier studies has been included. A parametric study of the impact of selected parameters on transport characteristics has been conducted. The simulations have shown that:

- Increasing sheet stretching ratio weakly accelerates the primary flow throughout the boundary layer whereas it more dramatically accelerates the secondary flow near sheet surface.

- Increasing magnetic field and inverse permeability parameter decrease both the primary and secondary velocity magnitudes and also the primary and secondary friction values, whereas they increase temperatures in the regime and reduce Nusselt number.

- Temperature is consistently reduced with increasing stretching sheet ratio whereas Nusselt number at the wall is enhanced.

- Temperature is strongly enhanced with greater radiative parameter whereas Nusselt number is reduced and there is no noticeable effect on either primary or secondary friction.

- With greater Sisko non-Newtonian power-law index the primary velocity and temperature are decreased whereas the secondary velocity is increased.

- Increasing both homogenous and heterogenous chemical reaction parameters is found to weakly and more strongly, respectively, reduce concentration magnitudes whereas greater Schmidt number enhances the concentration values in the bi-directional sheet.

- Primary skin friction is strongly reduced with increasing $A$ values (Sisko material parameter) whereas secondary skin friction is weakly reduced and Nusselt number is slightly increased.

- Increasing Sisko power-law index i.e. greater dilatant non-Newtonian behavior is found to elevate primary skin friction and reduce secondary skin friction whereas it initially increases and thereafter reduces Nusselt number. 
The present study has shown that ADM is a powerful approach for studying multi-physical high-temperature bi-axial stretching reactive materials processing flows of electro-conductive (magnetic) polymers. Future studies will explore alternative radiative flux models and also unsteady effects and will be communicated imminently.

\section{REFERENCES}

[1] Yamaguchi, H., Zhang, X.R., Higashi, S., Li, M., Study on power generation using electroconductive polymer and its mixture with magnetic fluid, J. Magn. Magn. Mat. 320 (2008) 14061411.

[2] Longo, G., Pompeo, G., Moreno, J.S., Panero, S., Girasole, M., Ronci, F., Cricenti, A., Morphological characterization of innovative electroconductive polymers in early stages of growth, Surface and Coatings Tech. 207 (2012) 286-292.

[3] Thévenot, J., Oliveira, H., Sandre, O., Lecommandoux., S., Magnetic responsive polymer composite materials, Chem. Soc. Rev. 42 (2013) 7099-7116.

[4] Pan, L., Yu, G., Zhai, D., Lee, H.R., Zhao, W., Liu, N., Wang, H., Tee, B.C.K., Shi, Y., Cui, Y., Bao, Z., Hierarchical nanostructured conducting polymer hydrogel with high electrochemical activity, Proc. Natl. Acad. Sci. USA, 109(24) (2012) 9287-9292.

https://doi.org/10.1073/pnas.1202636109

[5] Anwar Bég, O., Kuharat, S., Aneja, M., Sharma, S., Babaie, M., Numerical study of magnetic-bio-nano-polymer solar cell coating manufacturing flow, Int. Conf. on Innovative Appl. Energy (IAPE'19), Oxford, United Kingdom, 14-15 March (2019).

https://dx.doi.org/10.17501

[6] Maqbool, K., Anwar Bég, O., Ayesha, S., Shafaq, I., Analytical solutions for magnetohydrodynamic oscillatory rotating plate and channel flows in porous media using a fractional Burgers viscoelastic model, Euro. Phys. J. Plus. 131(2016) 140-157.

[7] Rahman, M.A., Uddin, M.J., Anwar Bég, O., Kadir, A., Influence of variable viscosity and thermal conductivity, hydrodynamic and thermal slips on magnetohydrodynamic micropolar flow: a numerical study, Heat Transfer-Asian Research (2019).

https://doi.org/10.1002/htj.21575 (17 pages)

[8] Ellahi, R., Zeeshan, A., Hussain, F., Abbas, T., Thermally charged MHD bi-phase flow coatings with non-Newtonian nanofluid and Hafnium particles along slippery walls, Coatings. 9 (2019) 300.

https://doi.org/10.3390/coatings9050300

[9] Sisko, A.W., The flow of lubricating greases, Indus. Eng. Chem. 50, 12 (1958)1789-1792.

[10] Malik, M.Y., Hussain, A., Salahuddin, T., Awais, M., Bilal, S., Khan, F., Flow of Sisko fluid over a stretching cylinder and heat transfer with viscous dissipation and variable thermal conductivity: A numerical study, AIP Adv. 6 (2016) 045118.

https://doi.org/10.1063/1.4948458 
[11] Khan, M., Abbas, Q., Duru, K., Magnetohydrodynamic flow of a Sisko fluid in annular pipe: a numerical study, Int. J. Numer. Meth. Fluids. 62 (2010) 1169-1180.

[12] Khan, M.K., Steady flow and heat transfer of a Sisko fluid in annular pipe, Int. J. Heat and Mass Transf. 53 (2010) 1290-1297.

[13] El-Dabe., N.T.M., Ghaly., A.Y., Sallam, S.N., Elagamy, K., Younis, Y.M., Peristaltic pumping of a conducting Sisko fluid through porous medium with heat and mass transfer, American J. Comput. Math. 5 (2015) 304-316.

[14] Zaman, A., Ali, N., Anwar Bég, O., Numerical study of unsteady blood flow through a vessel using Sisko model, Eng. Sci. Tech-An Int. J. 19 (2016) 538-547.

[15] Khan, M., Shehzad, A., On axisymmetric flow of Sisko fluid over a radially stretching sheet, Int. J. Non-Linear Mech. 47 (2012) 999-1007.

[16] Munir, A., Shahzad, A., Khan, M., Forced convective heat transfer in boundary layer flow of Sisko fluid over a nonlinear stretching sheet, PLoS One. 9(6) (2014) e100056.

https://doi.org/10.1371/journal.pone.0100056

[17] Shamshuddin, MD., Siva Reddy, S., Anwar Bég, O., Oscillatory dissipative conjugate heat and mass transfer in chemically reacting micropolar flow with wall couple stress: A finite element numerical study, Proc. IMechE Part E: J Proc. Mech. Eng. 233(1) (2017) 48-64.

[18] Rajesh, V., Anwar Bég, O., Sridevi, C., Finite difference analysis of unsteady MHD free convective flow over moving semi-infinite vertical cylinder with chemical reaction and temperature oscillation effects, J. Appl. Fluid Mech. 9 (2016) 157-167.

[19] Doh, D.H., Muthtamilselvan, M., Swathene, B., Ramya, E., Homogeneous and heterogeneous reactions in a nanofluid flow due to a rotating disk of variable thickness using HAM, Math. Comput. Simult. (2019).

https://doi.org/10.1016/j.matcom.2019.08.005

[20] Nadeem, S., Ullah, N., Khan, A.U., Akbar, T., Effect of homogeneous-heterogeneous reactions on ferrofluid in the presence of magnetic dipole along a stretching cylinder, Results in Phys. 7 (2017) 3574-3582.

[21] Ravi Kiran, G., Radhakrishnamacharya, G., Anwar Bég, O., Peristaltic flow and hydrodynamic dispersion of a reactive micropolar fluid: simulation of chemical effects in the digestive process, J. Mech. Med. Biol. 17 (2017) 1750013.1-1750013.17.

[22] Alshomrani, A.S., Ullah, M.Z., Effects of homogeneous-heterogeneous reactions and convective condition in Darcy-Forchheimer flow of carbon nanotubes, ASME J. Heat Transfer. 141(1) (2019) 012405.

https://doi.org/10.1115/1.4041553

[23] Abbas, Z., Sheikh, M., Numerical study of homogeneous-heterogeneous reactions on stagnation point flow of ferrofluid with non-linear slip condition, Chinese. J Chem Eng. 25(1) (2017) 11-17. 
[24] Chmielewski, A.G., Haji-Saeid, M., Ahmed, S., Progress in radiation processing of polymers, Nuclear Instruments and Methods in Physics Research Section B: Beam Interactions with Materials and Atoms, 236 (2005) 44-54.

[25] Bergman, T.L., Viskanta, R., Radiation heat transfer in manufacturing and materials processing, in Radiative Transfer-I, M.P. Menguc, Editor, pp. 13-39, Begell House, New York (1996).

[26] Lu, D., Ramzan, M., Bilal, M., Chung, J.D., Farooq, U., Tahir, S., On three-dimensional MHD Oldroyd-B fluid flow with nonlinear thermal radiation and homogeneous-heterogeneous reaction, J. Brazilian Soc. Mech. Sci. Eng. 40(8) (2018) 387.

https://doi.org/10.1007/s40430-018-1297-z

[27] Kumpaty, S.K., Roux, J.A., Investigation of thermal gradients in electrically annealed float glass, Transport Phenomena in Materials Processing and Manufacturing, M. Charmchi et al., Editors, HTD, AMSE, Newyork, 196 (1992) 153-159.

[28] Rozzi, J.C., Pfefferkorn, F.E., Incropera, F.P., Shin, Y.C., Transient, three-dimensional heat transfer model for the laser assisted machining of silicon nitride: I. Comparison of predictions with measured surface temperature histories, Int. J. Heat and Mass Transf. 43 (2000) 1409-1424.

[29] Anwar Bég, O., Ferdows, M., Bég, T.A., Ahmed, T., Wahiduzzaman, M., Alam, M.M., Radiative optically-dense magnetized transient reactive transport phenomena with cross diffusion and dissipation effects: numerical simulations, J. Taiwan Inst. Chem. Eng. 66 (2016) $12-26$.

[30] Hayat, T., Ullah, I., Alsaedi, A., Ahmad, B., Numerical simulation for homogeneousheterogeneous reactions in flow of Sisko fluid, J Brazilian Soc. Mech. Sci. Eng, 40 (2018) 73. https://doi.org/10.1007/s40430-018-0999-6

[31] Adomain, G., Solving Frontier Problems in Physics: The Decomposition Method, Kluwer, Dordrecht, USA (1994).

[32] Chaudhary, M.A., Merkin., J.H., A simple isothermal model for homogeneousheterogeneous reactions in boundary layer flow. II different diffusivities for reactant and auto catalyst, Fluid Dynam. Resear. 16 (1995) 335. https://doi.org/10.1016/0169-5983(95)90813-H.

[33] Khan, M., Ahmad, L., Khan, W.A., Numerically framing the impact of radiation on magneto nanoparticles for 3D Sisko fluid, J Brazilian Soc. Mech. Sci. Eng. 39 (2017) 44754487.

[34] Hayat T, Imtiaz M, Almezal S., Modeling and analysis for three-dimensional flow with homogeneous-heterogeneous reactions. AIP Adv. 5 (2015) 107209.

https://doi.org/10.1063/1.4933084. 
[35] Hayat, T., Muhammad, T., Ahmad, B., Shehzad, S.A., Impact of magnetic field in threedimensional flow of Sisko nanofluid with convective condition. J Magn. Magn. Mater. 413 (2016) $1-8$.

[36] Rahman, M.M., Locally similar solutions for hydromagnetic and thermal slip flow boundary layers over a flat plate with variable fluid properties and convective surface boundary condition, Meccanica. 46 (2011) 1127-1143.

[37] Sanjayanand, E., Khan, S.K., On heat and mass transfer in a viscoelastic boundary layer flow over an exponentially stretching sheet, Int. J. Therm. Sci. 45(2006) 819-828.

[38] Anwar Bég, O., Tripathi, D., Sochi, T., Gupta, P.K., Adomian decomposition method (ADM) simulation of magneto-bio-tribological squeeze film with magnetic induction effects, J. Mech. Med. Biol. 15 (2015) 1550072.1-1550072.23.

[39] Manzoor, N., Maqbool, K., Anwar Bég, O., Shaheen, S., Adomian decomposition solution for propulsion of dissipative magnetic Jeffrey biofluid in a ciliated channel containing a porous medium with forced convection heat transfer, Heat Transfer - Asian Research (Japan) (2018). https://doi.org/10.1002/htj.21394 (26 pages)

[40] Shamshuddin, MD., Mishra, S.R., Anwar Bég, O., Kadir., A., Adomian decomposition method (ADM) simulation of Von Kármán swirling bioconvection nanofluid flow, J. Central South Univ.-Sci. Tech. Mining and Metallurgy (2019), (17 pages)

\section{APPENDIX:}

The ADM power series expansions are

$$
\begin{aligned}
f(\eta)= & \eta+\frac{\eta^{2}}{2} p+\left(T_{1}+T_{7}\right) \eta^{3}+\left(T_{2}+T_{8}\right) \eta^{4}+\left(T_{3}+T_{9}\right) \eta^{5}+T_{10} \eta^{6}+T_{11} \eta^{7}+T_{12} \eta^{8} \\
g(\eta)= & \alpha \eta+\frac{\eta^{2}}{2} q+\left(T_{4}+T_{13}\right) \eta^{3}+\left(T_{5}+T_{14}\right) \eta^{4}+\left(T_{6}+T_{15}\right) \eta^{5}+T_{16} \eta^{6}+T_{17} \eta^{7}+T_{18} \eta^{8} \\
\theta(\eta)= & 1+\frac{1}{\gamma} r+r \eta+\left(T_{19}+T_{22}\right) \eta^{2}+\left(T_{20}+T_{23}\right) \eta^{3}+\left(T_{21}+T_{24}\right) \eta^{4}+T_{25} \eta^{5} \\
& +T_{26} \eta^{6}+T_{27} \eta^{7}+T_{28} \eta^{8}+T_{29} \eta^{9}+T_{30} \eta^{10} \\
\phi(\eta)= & \frac{1}{k_{2}} s+s \eta+\left(T_{31}+T_{35}\right) \eta^{2}+\left(T_{32}+T_{36}\right) \eta^{3}+\left(T_{33}+T_{37}\right) \eta^{4}+\left(T_{34}+T_{38}\right) \eta^{5} \\
& +T_{39} \eta^{6}+T_{40} \eta^{7}+T_{41} \eta^{8}+T_{42} \eta^{9}+T_{43} \eta^{10}+T_{44} \eta^{11}+T_{45} \eta^{12}
\end{aligned}
$$

The values of the notations $T_{i}{ }^{\prime} s, i=1-45$ are presented below:

$$
\begin{aligned}
& T_{1}=\frac{1+K+M^{2}}{6 A}, T_{2}=\left(-\frac{2 n}{n+1}+2 p-\alpha p+K p+M^{2} p\right) / 24 A, T_{3}=\left(-\frac{2 n}{n+1} p+p^{2}-\frac{p q}{2}\right) / 6 A, \\
& T_{4}=\frac{\alpha^{2}+M^{2}+K}{6 A}, T_{5}=\left(-\frac{2 n}{n+1}+\alpha q+\left(M^{2}+K\right) \alpha\right) / 24 A, T_{6}=\left(-\frac{2 n}{n+1} q+\frac{q^{2}}{2}\right) / 60 A,
\end{aligned}
$$




$$
\begin{aligned}
& T_{4}=\frac{n p^{n-1}}{A} T_{4}, T_{8}=\frac{n p^{n-1}}{A} T_{5}, T_{9}=\left(-60 n p^{n-1} T_{6}-\frac{12 n}{n+1} T_{1}+6 T_{1}-6 \alpha T_{1}+3\left(K+M^{2}\right) T_{1}\right) / 60 A, \\
& T_{10}=\left(-\frac{24 n}{n+1} T_{2}-\frac{6 n}{n+1} T_{1} p-\frac{2 n}{n+1} T_{1}+8 T_{2}+6 T_{1} p-12 \alpha T_{2}+3 T_{1} q+T_{4} p+4\left(K+M^{2}\right) T_{2}\right) / 120 A, \\
& T_{11}=\left(-\frac{40 n}{n+1} T_{3}-\frac{12 n}{n+1} T_{2} p+9 T_{2} p+10 T_{3}-20 \alpha T_{3}-6 T_{2} q+T_{5} p+5\left(K+M^{2}\right) T_{3}\right) / 210 A, \\
& T_{12}=\left(-\frac{22 n}{n+1} T_{3} p-10 T_{3} p-10 T_{3} q+T_{6} p\right) / 336 A, T_{13}=-\frac{p^{n-1}}{A} T_{4}, T_{14}=-\frac{p^{n-1}}{A} T_{5}, \\
& T_{15}=\left(-60 p^{n-1} T_{6}-\frac{12 n}{n+1} T_{4}-12 \alpha T_{4}+3\left(K+M^{2}\right) T_{4}\right) / 60 A, \\
& T_{16}=\left(-\frac{24 n}{n+1} T_{5}-\frac{6 n}{n+1} T_{4} p-\frac{2 n}{n+1} T_{1} q-4 T_{5} \alpha+2 T_{4} q+4\left(K+M^{2}\right) T_{5}\right) / 120 A, \\
& T_{17}=\left(-\frac{40 n}{n+1} T_{6}-\frac{12 n}{n+1} T_{5} p-\frac{2 n}{n+1} T_{2} q-10 T_{6} \alpha+2 T_{5} q+5\left(K+M^{2}\right) T_{6}\right) / 210 A, \\
& T_{18}=\left(-\frac{20 n}{n+1} T_{6} p-\frac{2 n}{n+1} T_{5} p-\frac{2 n}{n+1} T_{3} q-T_{6} q\right) / 336 A, T_{19}=-\frac{3}{2}\left(\theta_{w}-1\right) R r\left\{1+\left(\theta_{w}-1\right)\left(1+\frac{r}{\gamma}\right)\right\}, \\
& T_{20}=\left[-6\left(\theta_{w}-1\right) R r^{2}\left\{\left(\theta_{w}-1\right)\left(1+\frac{r}{\gamma}\right)+1\right\}-\frac{2 n}{n+1} r \operatorname{Pr}-r \operatorname{Pr} \alpha\right] / 6 \text {, } \\
& T_{21}=\left[-3\left(\theta_{w}-1\right)^{3} R r^{3}-\frac{n}{n+1} r \operatorname{Pr} p-2 r \operatorname{Pr} q\right] / 12 \text {, } \\
& T_{22}=\left[-2 R\left(1+\left(\theta_{w}-1\right)\left(1+\frac{r}{\gamma}\right)\right)^{3} T_{19}-6\left(\theta_{w}-1\right) R\left(1+\left(\theta_{w}-1\right)\left(1+\frac{r}{\gamma}\right)\right)^{2} T_{19}-3\left(\theta_{w}-1\right) R r\right] / 2, \\
& T_{23}=\left[-6 R\left(1+\left(\theta_{w}-1\right)\left(1+\frac{r}{\gamma}\right)\right)^{3} T_{20}-6 R\left(1+\left(\theta_{w}-1\right)\left(1+\frac{r}{\gamma}\right)\right)^{2} r T_{19}-6\left(\theta_{w}-1\right) R\left(1+\left(\theta_{w}-1\right)\left(1+\frac{r}{\gamma}\right)\right)^{2} T_{19}\right] / 6, \\
& T_{24}=\left[\begin{array}{l}
-12 R\left(1+\left(\theta_{w}-1\right)\left(1+\frac{r}{\gamma}\right)\right)^{3} T_{21}-18 R\left(1+\left(\theta_{w}-1\right)\left(1+\frac{r}{\gamma}\right)\right)^{2} r T_{20} \\
-3 R\left(1+\left(\theta_{w}-1\right)\left(1+\frac{r}{\gamma}\right)\right)^{2} r^{2} T_{19}-9\left(\theta_{w}-1\right) R\left(1+\left(\theta_{w}-1\right)\left(1+\frac{r}{\gamma}\right)\right)^{2} T_{20} \\
-12\left(\theta_{w}-1\right) R\left(1+\left(\theta_{w}-1\right)\left(1+\frac{r}{\gamma}\right)\right) r T_{19}-\frac{4 n}{n+1} \operatorname{Pr} T_{19}-2 \alpha \operatorname{Pr} T_{19}
\end{array}\right] / 12,
\end{aligned}
$$




$$
\begin{aligned}
& {\left[-36 R\left(1+\left(\theta_{w}-1\right)\left(1+\frac{r}{\gamma}\right)\right)^{3} r T_{21}-18 R\left(1+\left(\theta_{w}-1\right)\left(1+\frac{r}{\gamma}\right)\right)^{2} r T_{20}-2 R r^{3} T_{19}\right.} \\
& T_{25}=-12\left(\theta_{w}-1\right) R\left(1+\left(\theta_{w}-1\right)\left(1+\frac{r}{\gamma}\right)\right)^{2} T_{21}-18\left(\theta_{w}-1\right) R\left(1+\left(\theta_{w}-1\right)\left(1+\frac{r}{\gamma}\right)\right) r T_{20} / 20, \\
& -6\left(\theta_{w}-1\right) R r^{2} T_{19}-\frac{6 n}{n+1} \operatorname{Pr} T_{20}-\frac{2 n}{n+1} \operatorname{Pr} T_{1} p-\frac{2 n}{n+1} \operatorname{Pr} T_{19} r-3 \alpha \operatorname{Pr} T_{20} \\
& -\operatorname{Pr} p T_{19}-\operatorname{Pr} r T_{4} \\
& T_{26}=\left[\begin{array}{l}
-36 R\left(1+\left(\theta_{w}-1\right)\left(1+\frac{r}{\gamma}\right)\right) r^{2} T_{21}-18 R r^{3} T_{20}-24\left(\theta_{w}-1\right) R\left(1+\left(\theta_{w}-1\right)\left(1+\frac{r}{\gamma}\right)\right) T_{21} \\
-9\left(\theta_{w}-1\right) R r^{2} T_{20}-3\left(\theta_{w}-1\right)^{2} R r T_{19}-\frac{8 n}{n+1} \operatorname{Pr}_{21}-\frac{3 n}{n+1} \operatorname{Pr} T_{20}-\frac{2 n}{n+1} r \operatorname{Pr} T_{2} \\
-4 \alpha \operatorname{Pr} T_{21}-\frac{3}{2} \operatorname{Pr} T_{20}-\operatorname{Pr} r T_{5}
\end{array}\right] \\
& T_{27}=\left[\begin{array}{l}
-12 R r^{3} T_{21}-12\left(\theta_{w}-1\right) R r^{2} T_{21}-6\left(\theta_{w}-1\right)^{2} R T_{19} T_{20}-\frac{4 n}{n+1} \operatorname{Pr} T_{21}-\frac{2 n}{n+1} r \operatorname{Pr} T_{3} \\
-2 \operatorname{Pr} T_{21}-r \operatorname{Pr} T_{6}
\end{array}\right] / 42, \\
& T_{28}=\left(-3\left(\theta_{w}-1\right)^{3} R r T_{20}^{2}\right) / 56, T_{29}=\left(-6\left(\theta_{w}-1\right)^{2} R r T_{20} T_{21}\right) / 72, T_{30}=\left(-3\left(\theta_{w}-1\right)^{3} R r T_{21}^{2}\right) / 90 \text {, } \\
& T_{31}=\frac{1}{2} S c k_{1} s^{3} \frac{1}{k_{2}}\left(1-\frac{1}{k_{2}}\right), T_{32}=\left(-S c \frac{2 n}{n+1} s-S c \alpha s+S c k_{1} s^{3}\left(\left(1-\frac{1}{k_{2}}\right)\left(1-\frac{2}{k_{2}}\right)\right)\right) / 6, \\
& T_{33}=\left(-S c \frac{n}{n+1} p s-\frac{S c q s}{2}+S c k_{1} s^{3}\left(\frac{1}{k_{2}}-2\left(1-\frac{2}{k_{2}}\right)\right)\right) / 12, \quad T_{34}=S c k_{1} s^{3} / 20, T_{35}=\frac{S c k_{1}}{2 k_{1}} s, \\
& T_{36}=\left(-2 S c \frac{n}{n+1}+S c k_{1} s\right) / 6, T_{37}=\left[-\frac{n}{n+1} S c p-2 S c \alpha T_{31}+S c k_{1} T_{31}\left(1-\frac{1}{k_{2}} S\right)^{2}-S c k_{1} \frac{s}{k_{2}} T_{31}\right] / 12 \text {, } \\
& T_{38}=\left[S c \frac{2 n}{n+1} T_{1} s-3 S c \alpha s T_{32}-S c s T_{31}+S c k_{1}\left(T_{32}\left(1-\frac{1}{k_{2}} s\right)^{2}-T_{31} s^{2}\right)+S c k_{1}\left(-\frac{s}{k_{2}} T_{32}-s T_{31}\right)\right] / 20 \text {, } \\
& T_{39}=\left[S c \frac{2 n}{n+1} T_{2} s-4 S c s T_{33}-\frac{3}{2} S c s T_{32}+S c k_{1}\left(T_{33}\left(1-\frac{1}{k_{2}} s\right)^{2}-T_{32} s^{2}\right)+S c k_{1}\left(-\frac{s}{k_{2}} T_{33}-s T_{32}\right)\right] / 30 \text {, } \\
& T_{40}=\left[S c \frac{2 n}{n+1} T_{3} s-5 S c s T_{34}-2 S c s T_{33}+S c k_{1}\left(T_{34}\left(1-\frac{1}{k_{2}} s\right)^{2}-T_{33} s^{2}\right)+S c k_{1}\left(-\frac{s}{k_{2}} T_{34}-s T_{33}\right)\right] / 42 \text {, } \\
& T_{41}=\left[\frac{5}{2} S c T_{34} s-2 S c k_{1} T_{34}\left(1-\frac{1}{k_{2}} s\right) s+S c k_{1} T_{33} s^{2}-S c k_{1} s T_{34}\right] / 56,
\end{aligned}
$$




$$
\begin{aligned}
& T_{42}=\left[S c k_{1} T_{34} s^{2}+S c k_{1}\left(2 \frac{1}{k_{2}} s T_{32} T_{33}+s T_{32}^{2}\right)\right] / 72, T_{43}=S c k_{1}\left(\frac{s}{k_{2}} T_{33}^{2}+s T_{32} T_{34}\right) / 90, \\
& T_{44}=S c k_{1}\left(2 T_{33} T_{34} \frac{s}{k_{2}}+s T_{33}^{2}\right) / 110, T_{45}=S c k_{1}\left(T_{34}^{2} \frac{s}{k_{2}}+2 s T_{33} T_{34}\right) / 132
\end{aligned}
$$

